\title{
Konvergenz der Umweltpolitiken in Europa? \\ Der Einfluss internationaler Institutionen und der ökonomischen Integration*
}

\author{
Katharina Holzinger / Christoph Knill / Thomas Sommerer
}

\section{Einleitung}

Die Frage, ob die zunehmende transnationale Verflechtung zwischen Staaten zu einer Konvergenz ihrer Politiken, Institutionen, Rechtsordnungen oder gesellschaftlichen Strukturen führt, gehört zu den Kernfragen der Globalisierungsforschung (Guillèn 2001: 235). Während einige frühe Beiträge diese Frage positiv beantworteten ( $\mathrm{z}$. B. Meyer u. a. 1997), kamen andere zu dem Ergebnis, dass eine allgemeine Konvergenz eher nicht zu erwarten sei (z. B. Boyer 1996; Garrett 1999). In den letzten Jahren gibt es ein erneutes Interesse an der Erforschung transnationaler Politikkonvergenz. Stimmt es, dass die immer weiter zunehmende ökonomische und institutionelle Verflechtung zwischen Nationalstaaten zu einer wachsenden Ähnlichkeit der Politiken in verschiedenen Ländern führt? Oder ist die Suche nach Konvergenz „an impossible quest“ (Dimitrova/Streunenberg 2000: 201), da die Reaktion der einzelnen Länder auf globale oder europäische Herausforderungen stark von den bereits bestehenden nationalen Strukturen und Institutionen beeinflusst ist (vgl. z. B. Caparaso u. a. 2001; Héritier u. a. 2001; Knill 2001)?

Wir haben bisher noch kein hinreichendes Verständnis des Phänomens der Konvergenz von Politiken. Können wir überhaupt Konvergenz feststellen? Womit kann die Adaption ähnlicher Politiken zwischen Ländern erklärt werden? Unter welchen Bedingungen können wir eine Annäherung der Politiken erwarten, und unter welchen Bedingungen eher unterschiedliche Entwicklungen? Warum entsteht Konvergenz in bestimmten Politikfeldern, in anderen aber nicht? In der Literatur sind verschiedene Faktoren genannt worden, die die gemischten empirischen Befunde von sowohl Konvergenz als auch Persistenz und Divergenz erklären sollen. Trotzdem fehlt es immer noch an systematischen theoretischen und empirischen Untersuchungen, die den jeweiligen Erklärungsbeitrag dieser Faktoren deutlich machen könnten.

In diesem Aufsatz wollen wir einen Beitrag zur Schließung dieser Forschungslücken leisten. Wir untersuchen, ob und in welchem Ausmaß Konvergenz von Umweltpolitiken in Europa empirisch beobachtet und wodurch sie erklärt werden kann. Dazu analysieren wir die Entwicklung von 40 umweltpolitischen Maßnahmen in 24 Ländern

* Die in diesem Beitrag präsentierten Ergebnisse basiseren auf dem Forschungsprojekt ENVIPOLCON (Environmental Governance in Europe), das von der Europäischen Kommission im fünften Forschungsrahmenprogramm gefördert wurde (nähere Informationen unter http:// www.uni-konstanz.de/FuF/Verwiss/knill/projekte/envipolcon/project-homepage.php). Die Verfasser danken Heide Padberg für die hilfreiche Mitarbeit bei der Erstellung und Überarbeitung des Manuskriptes. 
zwischen 1970 und 2000. Wir konzentrieren uns dabei auf zwei Erklärungsfaktoren: die internationale ökonomische und institutionelle Verflechtung zwischen den Staaten.

Politikkonvergenz wird hier definiert als Zunahme der Ähnlichkeit einer oder mehrerer Dimensionen einer bestimmten Policy (z. B. die Existenz einer solchen Policy, die verwendeten Instrumente, die genaue Höhe einer Steuer oder eines Grenzwerts) innerhalb einer Gruppe von politischen Organisationseinheiten (i. d. R. Staaten) über einen festgelegten Zeitraum. Wir arbeiten also mit dem Konzept der $\sigma$-Konvergenz (siehe Heichel/Sommerer in diesem Band; Heichel u. a. 2005). Politikkonvergenz beschreibt also das Endergebnis von Prozessen des Polirikwandels in mehreren Ländern innerhalb eines bestimmten Zeitraums, die zu einer Annäherung führen (siehe Einleitungsbeitrag). Die Richtung der Konvergenzbewegung, d. h. Änderungen im umweltpolitischen Schutzniveau, ist nicht Gegenstand der Untersuchung.

Der Artikel ist wie folgt strukturiert: In Abschnitt 2 wird der theoretische Rahmen vorgestellt, während in Abschnitt 3 das Forschungsdesign, die Operationalisierung der verwendeten Variablen und die Datenerhebung erläutert werden. Die Ergebnisse der empirischen Untersuchung sind Gegenstand von Abschnitt 4. Generelle Schlussfolgerungen unserer Analyse werden in Abschnitt 5 präsentiert.

\section{Theorie und Hypothesen}

In der Literatur spielen internationale Faktoren eine wichtige Rolle für die Erklärung von länderübergreifender Politikkonvergenz (Bennett 1991; Dolowitz/Marsh 2000; Drezner 2001; Gilardi 2005; Hoberg 2001; Holzinger/Knill 2005; Simmons/Elkins 2004). Erstens beziehen sich diese Faktoren auf das Ausmaß der institutionellen Verflechtung zwischen den Staaten. In diesem Zusammenhang werden nicht nur Konvergenzeffekte betont, die durch die Harmonisierung nationaler Politiken aufgrund von internationaler oder supranationaler Gesetzgebung entstehen, sondern es wird auch auf die auch Effekte von Informationsaustausch und transnationaler Kommunikation innerhalb von institutionalisierten Netzwerken verwiesen. Zweitens wird der regulative Wettbewerb, der durch die wachsende ökonomische Integration in internationale Märkte entsteht, als wichtiger Kausalmechanismus für die gegenseitige Anpassung von Politiken zwischen Staaten identifiziert. Die Fokussierung auf die internationalen Faktoren bedeutet nicht, dass das durch nationale Faktoren entstehende Konvergenzpotenzial vernachlässigt wird. Die Ähnlichkeit oder Konvergenz nationaler Bedingungen könnte ähnliche politische Reaktionen und damit auch Konvergenz von Politiken auslösen (Bennett 1991: 231).

\subsection{Internationale Harmonisierung}

Der Mechanismus der internationalen Harmonisierung bezieht sich auf ein bestimmtes Ergebnis internationaler Kooperation, nämlich auf Konstellationen, in denen nationale Regierungen durch ihre Mitgliedschaft in internationalen Institutionen rechtlich verpflichtet sind, ähnliche Politiken und Programme zu übernehmen (Drezner 2001: 60; 
Hoberg 2001: 127; Martin/Simmons 1998: 743, siehe auch Holzinger/Knill in diesem Band).

Unter den Oberbegriff der internationalen Harmonisierung fassen wir auch die Konvergenzeffekte von Konditionalität (vgl. Schimmelfennig/Sedelmeier 2004), d. h. die Anpassung nationaler Politiken an internationale Vorgaben im Tausch gegen die Aufnahme in eine internationale Institution, wie etwa die Europäische Union (EU). Die Unterscheidung zwischen diesen beiden Mechanismen erfolgt üblicherweise so, dass Harmonisierung als freiwillige, Konditionalität als erzwungene Anpassung von Politiken betrachtet wird. Für den Bereich der europäischen Umweltpolitik ist diese Differenzierung wenig hilfreich. Es lässt sich kaum beurteilen, ob durch den umweltpolitischen acquis communautaire eine aus Sicht der EU-Beitrittskandidaten freiwillige oder erzwungene Einigung hervorgerufen wurde - der Beitritt erfolgt ja ebenso freiwillig wie die Unterwerfung unter eine gemeinsam in der EU beschlossene Regelung. Unabhängig davon gibt es bei den von uns untersuchten Politiken und Ländern keine Fälle, in denen die Anpassung einer Umweltpolitik eindeutig von außen erzwungen wurde.

Um die Auswirkungen internationaler Harmonisierung auf die Politikkonvergenz erfassen zu können, nehmen wir zunächst zwei Unterscheidungen vor. Internationale Institutionen unterscheiden sich erstens in der Art ihrer Harmonisierungseffekte und zweitens in ihrem Verbindlichkeitspotenzial. Aus beiden Unterscheidungen ergibt sich eine Differenzierung zwischen den Konvergenzeffekten der supranationalen Organisation EU und der aller anderen umweltbezogenen internationalen Institutionen (einschließlich internationaler Organisationen, Regime, Verträge und Protokolle).

Erstens unterscheiden wir zwischen Harmonisierungseffekten, die durch den Beitritt zu einer internationalen Institution entstehen, und solchen, die durch andauernde Mitgliedschaft in dieser Institution erwachsen. Erstere ergeben sich daraus, dass die beitretenden Mitglieder den Vertrag ratifizieren und die damit verbundenen Erfordernisse erfüllen müssen. Dies impliziert, dass Konvergenzeffekte durch Beitritt nur einmalig auftreten. Harmonisierungseffekte durch Mitgliedschaft hingegen entstehen, wenn die betroffene Institution über die Kompetenz und Autorität verfügt, laufend regulative Richtlinien für ihre Mitglieder zu erlassen, was zu ständig erneuerten Harmonisierungseffekten führt. Im Gegensatz zu den internationalen Institutionen bewirkt der supranationale Charakter der EU, dass Harmonisierungseffekte nicht nur durch Beitritt entstehen, sondern auch durch Mitgliedschaft, da die Institutionen der EU fortlaufend neue Gesetzesvorschläge entwickeln und erlassen. Im Gegensatz dazu basieren Harmonisierungseffekte im Fall von internationalen Institutionen ausschließlich auf dem Beitritt der Staaten.

Zweitens ist die Differenzierung zwischen EU und allen anderen Institutionen auch dadurch gerechtfertigt, dass diese Institutionen sich wesentlich in ihrem Verbindlichkeitspotenzial unterscheiden, das heißt in dem Ausmaß, in dem eine gesetzliche Verpflichtung letztendlich durchgesetzt werden kann. In der EU ist dieses „obligatorische Potenzial" deutlich besser entwickelt als in den anderen von uns untersuchten internationalen Institutionen. Dies gilt ungeachtet der Tatsache, dass letztere deutliche Unterschiede in ihrem Verpflichtungspotenzial und dadurch auch in dem Ausmaß aufweisen, in dem sie bei ihren Mitgliedern einen Politikwandel durchsetzen können. 
Vor dem Hintergrund dieser Differenzierungen ergeben sich drei Hypothesen zu den Auswirkungen internationaler Harmonisierung auf die Konvergenz nationaler Umweltpolitiken, die sich auf drei institutionelle Variablen beziehen:

(H1.1. - EU-Mitgliedschaft):

Wenn eine Gruppe von $n$ Ländern in $t_{0}$ Mitglieder der EU sind, konvergieren ihre Umweltpolitiken in den folgenden Perioden.

(H 1.2 - EU-Beitritt):

Wenn eine Gruppe von $n$ Ländern zwischen $t_{0}$ und $t_{1}$ der EU beitritt, so konvergieren die Umweltpolitiken dieser Länder in derselben Periode.

(H 1.3 - Beitritt zu Internationalen Institutionen):

Je größer die Zahl der internationalen Institutionen (und je größer ihr obligatorisches Potenzial), der eine Gruppe von $n$ Ländern zwischen $t_{0}$ und $t_{1}$ beigetreten ist, desto größer wird die Konvergenz zwischen ihren Umweltpolitiken in derselben Periode sein.

\subsection{Transnationale Kommunikation}

Internationale Institutionen erzeugen Konvergenzeffekte zwischen ihren Mitgliedstaaten nicht nur durch rechtlich verbindliche Regeln und Entscheidungen, sondern auch durch nicht-verbindliche Faktoren. Diese Erwartung ergibt sich aus verschiedenen theoretischen Argumenten, die im Bereich der Organisationssoziologie entwickelt wurden. Wie DiMaggio und Powell (1991) betont haben, tendieren häufig interagierende Organisationen dazu, mit der Zeit ähnliche Strukturen und Konzepte zu entwickeln. Politikkonvergenz entsteht oft dann, wenn Organisationen versuchen, ihre soziale Legitimität dadurch zu erhöhen, dass sie Praktiken umsetzen, die in ihrer institutionellen Umgebung positiv bewertet werden. Die Existenz internationaler Institutionen und Netzwerke spielt daher eine wichtige Rolle bei der Einführung neuer Politikmodelle und regulativer Innovationen über nationale Grenzen hinaus (Strang/Meyer 1993).

Es lassen sich zwei grundsätzliche Formen von Konvergenz durch Kommunikation und Lernen in internationalen Organisationen unterscheiden. Erstens können Staaten sich „mimetisch“ verhalten, wenn sie die erfolgreichen Politiken anderer Staaten für ihr Land zu kopieren versuchen. Zweitens spielt die Verbreitung professionellen Wissens über transnationale Netzwerke oder „epistemic communities“, die ähnliche Wahrnehmungen und Vorstellungen zu Problemen und den entsprechenden Lösungsansätzen im politischen Bereich haben (Haas 1992), eine wichtige Rolle bei der Verbreitung von Politikkonzepten durch gemeinsame Beratung und das Lernen von anderen.

Ausgehend von dieser Differenzierung nehmen wir an, dass Konvergenzeffekte transnationaler Kommunikation sich nicht sofort mit dem Beitritt einstellen, sondern mit der Dauer der Mitgliedschaft ansteigen. Da in dieser Hinsicht jedoch keine wesentlichen Unterschiede zwischen der EU und internationalen Institutionen bestehen, bedarf es an dieser Stelle keiner Differenzierung zwischen beiden Typen von Institutionen. Allerdings ist zu berücksichtigen, dass internationale und supranationale Institutionen wichtige Unterschiede im Hinblick auf ihr „kommunikatives Potenzial“ aufwei- 
sen. Dieses Potenzial variiert mit der Häufigkeit und dem Umfang der Interaktionen im Rahmen der jeweiligen Institution (Kern 2000: 267).

(H 2 - Institutionelle Mitgliedschaft):

Je größer die Zahl der Institutionen (und je größer ihr kommunikatives Potenzial), in der eine Gruppe von $n$ Ländern in $\mathrm{r}_{0}$ gleichzeitig Mirglied ist, desto stärker werden ihre Umweltpolitiken in den nachfolgenden Perioden konvergieren.

\subsection{Regulierungswettbewerb}

Den Theorien des Regulierungswettbewerbs zufolge tendieren Länder, die unter Konkurrenzdruck stehen dazu, ihre Politiken aneinander anzupassen. Regulativer Wettbewerb setzt wirtschaftliche Integration zwischen Ländern und das Konkurrenzprinzip voraus. Die steigende Integration europäischer und globaler Märkte und die damit verbundene Abschaffung von Beschränkungen für den Austausch von Gütern, Dienstleistungen und Kapital setzt die Staaten einem starken Druck aus, bestehende nationale Regulierungen so zu verändern, dass ihre wirtschaftliche Wettbewerbsfähigkeit gesichert wird. Wettbewerbsdruck auf die Regierungen entsteht durch Lobbying und die (potentielle oder tatsächliche) Drohung von Seiten wirtschaftlicher Akteure, ihre Aktivitäten in andere Länder zu verlegen. Dies veranlasst, so die Theorie, nationale Regierungen dazu, ihre Politiken entsprechend zu verändern.

In der Literatur zum Thema gibt es eine ausgedehnte Debatte darüber, ob und wann diese Anpassungen mit dem Absenken oder der Erhöhung des umweltpolitischen Schutzniveaus einhergehen (Drezner 2001: 57-59; Holzinger 2003; Scharpf 1997; Simmons/Elkins 2004; Vogel 1995). Da der Schwerpunkt unserer Analyse aber auf dem Grad und nicht auf der Richtung der Konvergenz liegt, ist es ausreichend anzunehmen, dass Theorien des regulativen Wettbewerbs für Länder, die dem internationalen Wettbewerbsdruck ausgesetzt sind, einen Anstieg der Politikkonvergenz vorhersagen, unabhängig von dem regulativen Niveau, auf dem diese Konvergenz auftritt.

Je mehr ein Land durch wirtschaftliche Integration entsprechendem Wettbewerbsdruck ausgesetzt ist, desto wahrscheinlicher ist es, dass seine Politiken mit denen anderer Länder konvergieren, die sich in einer ähnlichen Situation befinden. Der Grad der Konvergenz hängt also davon $a b$, in welchem Ausmaß die Länder dem Wettbewerbsdruck ausgesetzt sind. In diesem Zusammenhang werden üblicherweise die Außenhandelsoffenheit eines Landes oder der zwischenstaatliche Handel als Indikatoren für die wirtschaftliche Vernetzung zwischen Ländern benutzt (z. B. Simmons/Elkins 2004).

(H 3 - Bilateraler Handel):

Je mehr Handel zwischen einer Gruppe von $\mathrm{n}$ Ländern in Periode $\mathrm{t}_{0}$ betrieben wird, desto mehr konvergieren ihre Umweltpolitiken zwischen $t_{0}$ und $t_{1}$.

Zwei Differenzierungen sind hier noch zu beachten. Erstens entsteht in oder zwischen Ländern ohne Marktwirtschaft selbst in Konstellationen mit hoher wirtschaftlicher Integration keinerlei Konkurrenzdruck. Zweitens werden Anpassungen nur für handelsrelevante Politiken erwartet, so zum Beispiel für umweltbezogene Produkt- oder Prozess- 
standards. Wir erwarten keine Konvergenz bei Politiken, die keinerlei Bezug zum Handel haben. Dies ist beispielsweise der Fall beim Naturschutz.

\subsection{Weitere Faktoren}

Politikkonvergenz wird nicht nur durch internationale Faktoren hervorgerufen, sondern könnte auch das Ergebnis paralleler nationaler Problemkonstellationen sein, die ähnliche Lösungsansätze provozieren. Im Bereich der Umweltpolitik könnten eine Reihe von Faktoren eine solche Rolle spielen (vgl. Holzinger/Knill in diesem Band).

Erstens gibt es Faktoren, die den Transfer und die Emulation von Politiken vereinfachen und daher mit der Zeir zu einem Anstieg der Ähnlichkeit transnationaler Politiken führen. Hier ist vor allem die kulturelle Ähnlichkeit zwischen Ländern, z. B. in Bezug auf Sprache, Religion oder geographische Nähe, zu nennen. Diese Gegebenheiten sind auch dann wirksam, wenn es keine starke institutionelle Integration zwischen Ländern gibt (vgl. Holzinger/Knill 2005; Simmons/Elkins 2004). Ein zweiter Faktor bezieht sich auf das Verhältnis zwischen dem wirtschaftlichen Entwicklungsniveau eines Landes und der Strenge seiner Umweltpolitik, so wie es in der „Kuznets-Kurve“ für die Umwelt ausgedrückt wird (Panayotou 1993; Stern/Common 2001). Diese zeigt, dass das Niveau der Umweltverschmutzung mit dem Bruttoinlandsprodukt (BIP) bis zu einem bestimmten Punkt steigt, danach aber wieder sinkt, weil sich in reichen Ländern die politische Nachfrage nach Schutz der Umwelt verstärkt. Drittens kann die Konvergenz nationaler Politiken das Ergebnis ähnlicher Nachfrage nach einer umfassenden und stringenten Umweltpolitik sein, die zum Beispiel durch die Existenz grüner Parteien oder Umweltorganisationen zum Ausdruck kommt. Viertens wird von ähnlichem Problemdruck (z. B. ähnlichen Luftverschmutzungswerten oder ähnlicher Bevölkerungsdichte) erwartet, dass er ähnliche Lösungsmodelle hervorruft. Schließlich ist davon auszugehen, dass Länder, deren Umweltpolitiken schon Ähnlichkeiten aufweisen, sich in späteren Perioden schneller annähern werden als Länder, zwischen denen es zunächst keine oder geringe Ähnlichkeiten gibt. Theorien der Politikkonvergenz durch transnationale Kommunikation und Lernen weisen darauf hin, dass der Grad der schon existierenden Ähnlichkeit Einfluss auf den Grad der in Zukunft zu erwartenden Konvergenz haben. Man kann aber auch annehmen, dass es hier einen „Sätrigungseffekt" gibt: Wenn eine Gruppe von Ländern sich in ihrer Umweltpolitik schon sehr ähnelt, ist das Potenzial für weitere Konvergenzeffekte gering. Auf der Grundlage dieser Annahmen können folgende Hypothesen formuliert werden:

(H 4.1 - Kulturelle Ähnlichkeit):

Je höher die kulturelle Ähnlichkeit zwischen einer Gruppe von n Ländern, desto eher werden ihre Umweltpolitiken konvergieren.

(H 4.2 - Einkommensniveau):

Je höher das Einkommensniveau in einer Gruppe von $n$ Ländern und je ähnlicher dieses in $t_{0}$ ist, desto eher konvergieren ihre Umweltpolitiken in den nachfolgenden Perioden. 
(H 4.3 - Politische Nachfrage):

Je höher die politische Nachfrage für Umweltschutz in einer Gruppe von n Ländern und je ähnlicher diese in Periode $t_{0}$ ist, desto eher konvergieren ihre Umweltpolitiken in den nachfolgenden Perioden.

(H 4.4 - Problemdruck):

Je höher der Problemdruck durch Umweltverschmutzung in einer Gruppe von $\mathrm{n}$ Ländern und je ähnlicher dieser in Periode $t_{0}$ ist, desto eher konvergieren ihre Umweltpolitiken in den nachfolgenden Perioden.

(H 4.5 - Bestehende Politikähnlichkeit):

Je ähnlicher die Umweltpolitiken in einer Gruppe von $\mathrm{n}$ Ländern in Periode $\mathrm{t}_{0}$ sind, desto eher konvergieren ihre Umweltpolitiken in den nachfolgenden Perioden, solange der erreichte Grad an Ähnlichkeit noch nicht sehr hoch ist.

\section{Daten und Variablen}

Ziel dieses Beitrages ist nicht nur die Analyse, ob und in welchem Umfang die Umweltpolitiken der betrachteten Länder konvergieren, sondern auch die Erklärung dieser Entwicklungen auf der Basis der oben vorgestellten Mechanismen. In diesen Abschnitt wird zunächst erläutert, wie die abhängige Variable (Konvergenz der Umweltpolitik) gemessen wird; daran anschließend wird die Operationalisierung der Kausalmechanismen vorgenommen.

\subsection{Die abhängige Variable}

\section{Auswabl der Politikfelder, der Länder und des Zeitraums}

Die Umweltpolitik der untersuchten Länder wird durch eine Auswahl von 40 umweltpolitischen Maßnahmen (im Folgenden als Policies bezeichnet) repräsentiert. Diese Auswahl kann schon allein deshalb nicht repräsentativ sein, weil es keine abgeschlossene Grundgesamtheit umweltpolitischer Maßnahmen gibt. Wir können also streng genommen nur Aussagen über diese 40 Policies machen. Die Auswahl umfasst aber wichtige Policies aus allen Umweltmedien und auch Querschnittsregelungen (vgl. Tabelle A1 im Anhang) und ist insofern durchaus ausgewogen. Wir benutzen Output-Daten, also die rechtlichen Regelungen, nicht deren Wirkungen (Outcome). Das hat den Grund, dass die oben eingeführten Kausalmechanismen auf Mikrotheorien basieren, in deren Zentrum jeweils die Entscheidung von Regierungen steht, Policies anzupassen oder nicht.

Für diese Policies wurden Daten zu drei Dimensionen erhoben:

(1) Politikpräsenz: Verfügt ein Land über eine entsprechende Politik oder nicht?

(2) Politikinstrumente: Welche dominanten Instrumente kommen im Rahmen dieser Policy zur Anwendung (z. B. Pläne, Grenzwerte, Steuern)? 
(3) Regulierungsniveau: Wie ist die konkrete Spezifikation der Instrumente (wie hoch sind etwa festgelegte Grenzwerte oder Steuersätze)?

Nicht bei jeder der 40 Policies lassen sich alle drei Dimensionen abfragen: Bei der Frage nach dem Vorliegen eines Umweltplans etwa, kann weder nach dem Instrument noch nach dem Regulierungsniveau gefragt werden. Nur für die erste Dimension liegen Daten zu allen 40 Policies vor. Daten zur Dimension der Instrumente konnten für 28 Policies erhoben werden, während sich für 21 Policies Regulierungsniveaus messen ließen.

In einer umfassenden Variable sind alle 40 Policies des Datensatzes enthalten. Zusätzlich werden aus theoretischen Gründen die folgenden Teilgruppen von Variablen unterschieden: Erstens unterscheiden wir zwischen handelsrelevanten und nicht-handelsrelevanten Policies. Entsprechend der Theorie des Regulierungswettbewerbs, sollten die Konvergenzeffekte bei handelsrelevanten Policies größer sein. Zweitens unterscheiden wir obligatorische und nicht-obligatorische Policies. Obligatorisch sind solche Policies, für die eine verbindliche internationale Regelung besteht, z. B. eine EU-Richtlinie. Bei obligatorischen Policies sollten die Konvergenzeffekte durch internationale Harmonisierung deutlicher ausgeprägt sein als für nicht-obligatorische Policies. Für letztere kann davon ausgegangen werden, dass es sich bei Konvergenzeffekten um Effekte transnationaler Kommunikation handelt. ${ }^{1}$

Um Konvergenz zu messen, vergleichen wir zunächst den Grad der Ähnlichkeit von Politiken zu vier verschiedenen Zeitpunkten (1970, 1980, 1990, und 2000). Das Jahr 1970 ist die Geburtsstunde der nationalen und internationalen Umweltpolitik. Das Jahr 2000 war das letzte Jahr vor Beginn der Datenerhebung, für das vollständige Daten zur Verfügung standen. Dazwischen messen wir im Abstand eines Jahrzehnts. Dies hat zwei Gründe: Zum einen wäre die Erhebung von Zeitreihendaten mit einem unvertretbaren Aufwand verbunden gewesen. Zum anderen wandeln sich nationale Politiken nicht sehr häufig, so dass die Erstellung einer vollständigen Zeitreihe kaum zusätzliche Varianz erbracht hätte.

Die 24 untersuchten Länder umfassen zunächst die Mitgliedstaaten der EU-15 in 2000 (außer Luxemburg). Über den Untersuchungszeitraum von dreißig Jahren werden damit die verschiedenen Beitrittswellen erfasst (1973, 1981/1986, 1995). Dazu kommen im Jahr 2004 und 2007 beigetretene Staaten, welche im Jahr 2000 Kandidatenstatus hatten (Polen, die Slowakei, Ungarn, Bulgarien und Rumänien), Länder, die zwar nicht Mitglied sind, aber enge Beziehungen mit der EU unterhalten (Norwegen und die Schweiz) und Länder, die institutionell nicht in die EU integriert sind oder auch keine sonstigen engen Beziehungen unterhalten (USA, Japan und Mexiko). Diese Differenzierung erlaubt es, den Einfluss der EU-Mitgliedschaft systematisch abzuschätzen.

Für die Erhebung der Daten wurde ein Fragenkatalog entwickelt, der für jedes Land von nationalen umweltpolitischen Experten, meist Juristen und Politikwissen-

1 Beim Kodieren der Daten berücksichtigen wir die Tatsache, dass dieselbe Politik sich von einer nicht-obligatorischen zu einer obligatorischen wandeln kann, weil sie erst später von einer internationalen Institution als obligatorische Regelung verabschiedet wurde (vgl. Tabelle Al im Anhang). 
schaftlern, beantwortet wurde. ${ }^{2}$ Die Experteninformationen wurden anschließend auf Vollständigkeit und Korrektheit geprüft und verglichen.

\section{Die Messung von Politikähnlichkeit: Der Paarvergleich}

Unser Messinstrument für Konvergenz basiert auf einem Ansatz, in dem Länderpaare die Analysegrundlage bilden. ${ }^{3}$ Konvergenz zeigt sich dabei als eine Zunahme der Ähnlichkeit von Policies zweier Länder in einen bestimmten Zeitraum. Der Paarvergleich bietet für unsere Zielsetzung mehrere Vorteile (vgl. Holzinger 2006). Erstens ist der Paarvergleich gegenüber herkömmlichen Verfahren zur Messung der $\sigma$-Konvergenz der direktere Weg, nationale Politikveränderungen zu vergleichen. So ist es die gemeinsame Mitgliedschaft eines Paares von Ländern in einer internationalen Institution oder der bilaterale Handel, die die Konvergenz dieser Länder durch internationale Harmonisierung, transnationale Kommunikation oder Regulierungswettbewerb hervorrufen. Zweitens eröffnet der Paarvergleich im Gegensatz zu klassischen Ansätzen, die $\sigma$-Konvergenz im Aggregat des gesamten Ländersamples messen (z. B. mit dem Variationskoeffizienten), die Möglichkeit eine Konvergenzvariable als Explanandum in einem quantitativen Modell zu verwenden, die es sogar erlaubt, jede Konvergenz- oder Divergenzbewegung zu erfassen. Während die Aggregatmaße nur die gesamte Ländergruppe zu einem Zeitpunkt beschreiben und somit nicht als abhängige Variable in einem Erklärungsmodell benutzt werden können, geht beim Paaransatz die Information für die Entwicklung jedes Paares in jeder Dekade in das Modell ein. Drittens kann der Paaransatz für kategorische und metrische Daten verwendet werden, während der Variationskoeffizient nur bei metrischen Daten eingesetzt werden kann. Der Paarvergleich ist also geeignet, die verschiedenen Policy-Dimensionen in unserem Datensatz (Politikpräsenz, Instrumente und Regulierungsniveaus) in einer Variable zu erfassen.

Der Paarvergleich hat jedoch auch Schwächen. Methodisch ist er nicht völlig unabhängig von der Zusammensetzung der Stichprobe, denn der Wert eines bestimmten Länderpaares kann durch die Werte anderer Länderpaare bestimmt werden. Diesen Nachteil haben jedoch auch andere Ansätze, weil der Vergleich mit einem Referenzpunkt, der durch die Zusammensetzung der Stichprobe definiert ist, dem Konzept der Konvergenz immanent ist. Der Paarvergleich erhöht gleichzeitig die Anzahl der Fälle von 24 Ländern auf 276 Länderpaare. Da die Anzahl der ursprünglich zu untersuchenden Subjekte unverändert bleibt, könnte dieser Anstieg zu einer Unterschätzung des Standardfehlers in den quantitativen Modellen führen. Dies muss bei der Interpretation berücksichtigt werden. Wägt man jedoch Schwächen und Stärken ab, bietet der Paarvergleich die Möglichkeit eines einfachen, direkten und leistungsfähigen Zugangs zur Untersuchung von Konvergenz.

\section{Berechnung von Konvergenz}

Für die Konvergenzmessung wird der ursprüngliche Datensatz auf Länderbasis zunächst in einen dyadischen Datensatz umgeformt, der die Angaben für jedes Länder-

2 Da es sich um einen erheblichen Aufwand handelte, erhielten die Experten einen Werkvertrag.

3 In der Konvergenzforschung ist die Verwendung von Länderpaaren relativ neu (ein Beispiel ist Barrios u. a. 2003). 
paar enthält. Doppelte Paare (Länder A und B, Länder B und A) werden ausgeschlossen, so dass jedes Paar nur einmal vorkommt. Die Ähnlichkeitswerte werden wie folgt berechnet: Für die Dimensionen der Politikpräsenz und der Instrumente ist die Feststellung der Ähnlichkeit trivial: der Wert "1“ bedeutet, dass die Länder A und B dieselbe Politik verfolgen oder dieselben Instrumente verwenden, während „ 0 “ bedeuter, dass diese Parameter unterschiedlich sind. Für die Dimension des Regulierungsniveaus verwenden wir einen standardisierten metrischen Wert zwischen 0 und 1 auf Grundlage der Grenzwerte der Länder A und B. Dies führt zu einer Ähnlichkeitsskala zwischen 1 (die Grenzwerte sind identisch) und 0 (das Länderpaar, dessen Werte am weitesten auseinander liegen). Für alle anderen Werte wird die graduelle Ähnlichkeit auf der Basis der Distanz zwischen den beiden am weitesten auseinander liegenden Werten berechnet. Um zu vermeiden, dass Ausreißer das Ergebnis verfälschen, benutzen wir die Distanz zwischen dem 90 Prozent- und dem 10 Prozent-Quantil der empirischen Werteverteilung.

Addiert man die Ähnlichkeitswerte für alle Dimensionen, ergibt sich eine Ähnlichkeitsskala von 0 bis 89 Punkten ( 40 plus 28 plus 21) für die generelle Variable, die alle Policies enthält. Aus Gründen der besseren Vergleichbarkeit mit anderen Skalen und zur leichteren Interpretation werden die Punkt- in Prozentwerte umgewandelt, wobei das Maximum von 89 Punkten einem Wert von 100 Prozent Ähnlichkeit der Umweltpolitiken entspricht.

Die Ähnlichkeitswerte können leicht in Konvergenzwerte umgewandelt werden. Konvergenz wird gemessen über die Veränderungen des Prozentsatzes absoluter Ähnlichkeit zwischen $t_{0}$ und $t_{1}$. Die Policies, für die Länderpaar $A B$ schon in Periode $t_{1}$ vollständige Ähnlichkeit gezeigt hat, werden bei der Berechnung der Konvergenzwerte für die folgenden Perioden nicht weiter berücksichtigt. Dieses Vorgehen erfasst die Saturierungseffekte; andernfalls wäre die Folge eine Unterbewertung von Konvergenz. Die genaue Angaben zur Berechnung befinden sich in Tabelle A2 im Anhang.

\subsection{Unabhängige Variablen}

\section{Internationale Harmonisierung}

Entsprechend unseren theoretischen Ausführungen unterscheiden wir drei Variablen, um die Effekte internationaler Harmonisierung zu analysieren: „EU-Mitgliedschaft“, "EU-Beitritt", und „Beitritt zu internationalen Institutionen“. Für die gemeinsame „EU-Mitgliedschaft“ und den gemeinsamen „EU-Beitritt“ benutzen wir eine DummyVariable. Als Datum des Beitritts nehmen wir den Beginn der Beitrittsgespräche, weil die Beitrittskandidaten zu diesem Zeitpunkt meist schon beginnen, den acquis commuautaire zu implementieren. Für die dritte Variable haben wir Daten über die Mitgliedschaft von Ländern in 34 internationalen Organisationen und Regimen gesammelt. Die gemeinsame Mitgliedschaft in diesen Organisationen wird gewichtet mit jeweils einem Indikator für den Umfang der umweltbezogenen Aktivitäten der jeweiligen Organisation und einem Indikator für deren obligatorisches Potenzial (siehe Tabelle A3 in Anhang). 


\section{Transnationale Kommunikation}

Diese Variable erfasst die gemeinsame Mitgliedschaft in allen internationalen Institutionen einschließlich der EU. Die Mitgliedschaftsdaten werden wiederum gewichtet mit dem Umfang der Aktivitäten und dem kommunikativen Potenzial der Institutionen sowie der Dauer der Mitgliedschaft eines Landes in der jeweiligen Institution. Der Indikator für das kommunikative Potenzial erfasst z. B. die Häufigkeit der Treffen und die Zahl der Umweltgremien in den Organisationen. Die Gewichtung mit der Dauer der Mitgliedschaft beruht auf der oben ausgeführten Annahme, dass Kommunikationseffekte mit der Dauer der Mitgliedschaft steigen (siehe Tabelle A3).

\section{Regulativer Wettbewerb}

Für die Messung der ökonomischen Verflechtung eines Länderpaares verwenden wir Daten über bilaterale Handelsbewegungen. Während die Integration in den Welthandel normalerweise durch den Index der Außenhandelsoffenheit erfasst wird, ist die bilaterale Analyse der Handelsbewegungen für den Paarvergleich adäquater. Genauso wie für die Handelsoffenheit wird auch das bilaterale Handelsvolumen auf die Größe der nationalen Wirtschaft bezogen. Wir benutzen das BIP des kleineren Marktes, um die Abhängigkeit vom internationalen Handel besser zu erfassen. Wir berücksichtigen dabei nur Handelsbewegungen zwischen Marktwirtschaften, da von anderen Ökonomien (z. B. den osteuropäischen Ländern vor 1989) keinerlei Effekte erwartet werden, für die Theorien des regulativen Wettbewerbs von Bedeutung wären.

\section{Kontrollvariablen}

Für die Messung kultureller Ähnlichkeit wurde ein Index aus Religion, Sprache und geographischer Nähe entwickelt, mit dem Ähnlichkeitswerte für jedes Länderpaar berechnet werden können. Für die Bewertung der Einkommensniveaus benutzen wir die Differenz des Pro-Kopf-Einkommens eines Länderpaares und ihre absoluten Einkommenswerte. Für die politische Nachfrage nach einer umfassenden und stringenten Umweltpolitik wird der Einfluss grüner Parteien über Wahlerfolg, Mitgliedschaft im Parlament und Mitgliedschaft in der Regierung gemessen. Einen hohen Wert erhält ein Länderpaar dann, wenn grüne Parteien in beiden Ländern ähnlich einflussreich sind. Umweltpolitischer Problemdruck wird mit zwei Variablen getestet, $\mathrm{CO}_{2}$-Emissionen und Bevölkerungsdichte. Um den gemeinsamen Druck eines Länderpaares zu messen, werden jeweils der niedrigere Wert für Emissionen und Bevölkerungsdichte berücksichtigt. Um schließlich die schon bestehende Politikähnlichkeit zu messen, verwenden wir eine Variable, die das Niveau der Ähnlichkeit einzelner Policies zwischen Länderpaaren in der vorhergehenden Periode erfasst. Einzelheiten zu den Indikatoren finden sich in Tabelle A3; die deskriptive Statistik zu den unabhängigen Variablen findet sich in Tabelle A4 (jeweils im Anhang). 


\section{Ergebnisse}

\subsection{Deskriptive Ergebnisse: Gibt es Konvergenz?}

Einen Überblick über unsere empirischen Ergebnisse zur Ähnlichkeit und Konvergenz der Policies gibt Tabelle 1. Sie enthält in der linken Hälfte für alle Policies und für alle Teilgruppen die Mittelwerte der Ähnlichkeit für 276 Länderpaare (in Prozent) zu den vier Zeitpunkten (1970, 1980, 1990 und 2000), während in der rechten Hälfte die Veränderungsraten der Ähnlichkeit, also die Konvergenz für die drei Perioden angegeben ist. Die letzte Spalte gibt die Konvergenz für den Durchschnitt der drei Perioden an.

Erstens zeigen die Ergebnisse, dass die Ähnlichkeit für alle Policies zwischen 1970 und 2000 deutlich gewachsen ist (von 0.04 auf 0.56 ). Dies gilt gleichermaßen für alle Teilgruppen der Policies. Zweitens sind die Konvergenzeffekte in den 1990er Jahren am höchsten (0.37). Drittens sind die Ähnlichkeitseffekte im Hinblick auf die Dimension Politikpräsenz am deutlichsten ausgeprägt (in 2000: 0.65), gefolgt von den Instrumenten (0.61) und den Regulierungsnvieaus (0.33). Ein ähnliches Bild ergibt sich für die Analyse der Konvergenz (im Durchschnitt der Gesamtperiode: 0.27; 0.24; 0.12). Bei den Teilgruppen zeigt sich, viertens, eine stärkere Konvergenz handelsrelevanter Policies (im Vergleich zu nicht handelsrelevanten Maßnahmen) und obligatorischer Policies (im Vergleich zu nicht-obligatorischen Maßnahmen).

Tabelle 1: Ähnlichkeit von Politiken und Politikkonvergenz: Mittelwerte in Prozent für 276 Länderpaare

\begin{tabular}{|l|cccc|cccc|}
\hline & \multicolumn{4}{|c|}{ Politikähnlichkeit } & \multicolumn{5}{c|}{ Politikkonvergenz $^{1}$} \\
\hline & 1970 & 1980 & 1990 & 2000 & $1970 s$ & $1980 s$ & $1990 s$ & $\varnothing$ \\
\hline Paarvergleich alle Policies (89) & 0.04 & 0.14 & 0.29 & 0.56 & 0.10 & 0.18 & 0.37 & 0.22 \\
\hline Politikpräsenz (40) & 0.03 & 0.12 & 0.30 & 0.65 & 0.09 & 0.20 & 0.51 & 0.27 \\
Politikinstrumente (28) & 0.06 & 0.21 & 0.38 & 0.61 & 0.16 & 0.21 & 0.36 & 0.24 \\
Regulierungsniveau (21) & 0.00 & 0.07 & 0.17 & 0.33 & 0.06 & 0.10 & 0.18 & $\mathbf{0 . 1 2}$ \\
\hline Handelsrelevante Policies (63) & 0.04 & 0.17 & 0.35 & 0.64 & 0.14 & 0.22 & 0.45 & 0.27 \\
Nicht-handelsrelevante Policies (26) & 0.04 & 0.07 & 0.16 & 0.38 & 0.03 & 0.10 & 0.27 & 0.13 \\
\hline Obligatorische Policies (9/24/36/45) & 0.04 & 0.26 & 0.45 & 0.67 & 0.23 & 0.34 & 0.49 & 0.35 \\
Nicht-obligatorische Policies (80/65/53/44) & 0.05 & 0.09 & 0.19 & 0.45 & 0.06 & 0.09 & 0.30 & $\mathbf{0 . 1 5}$ \\
\hline
\end{tabular}

1 korrigiert für Saturierungseffekte.

\subsection{Analysemethode}

Die von uns verwendeten Modelle basieren auf einem gepoolten Datensatz, in dem die Konvergenz für drei Querschnittsmodelle je für die 1970er, 1980er und 1990er Jahre zusammengefasst wurde. Jedes dieser drei Querschnittsmodelle enthält Beobachtungen von 276 Länderpaaren. Wie oben beschrieben bezieht sich die abhängige Variable in allen Modellen auf den Grad der Veränderung der Politikähnlichkeit zwischen Beginn und Ende der jeweiligen Dekade. Die Veränderung wird in Prozent angegeben. 
Wir verwenden gewöhnliche OLS-Regressionsmodelle. Um unsere Hauptfrage nach dem Einfluss der internationalen Antriebskräfte für die Konvergenz der Umweltpolitiken beantworten zu können, gehen wir schrittweise vor (siehe Tabelle 2). Wir beginnen mit einem Regressionsmodell (Modell 1), das lediglich das Erklärungspotenzial der Variablen für internationale Harmonisierung aufzeigt (EU-Beitritt, EU-Mitgliedschaft, Beitritt zu internationalen Institutionen). In den Modellen 2 und 3 werden die Variablen für transnationale Kommunikation (Mitgliedschaft in internationalen Institutionen) und Regulierungswettbewerb (bilateralen Handel) ergänzt. Modell 4 zeigt das alleinige Erklärungspotenzial aller Kontrollvariablen. Modell 5 integriert alle Variablen, einschließlich einer Zeitvariable.

Die Modellvariablen sind jedoch nicht völlig unabhängig voneinander: So ist z. B. davon auszugehen, dass EU-Mitgliedschaft mit einem höheren Ausmaß an Handelsverflechtung einhergeht. Wir haben es also mit Multikollinearität der unabhängigen Variablen zu tun. Zwar kann der Ansatz der schrittweisen Einführung der Variablen hier schon Hinweise geben, wie die Koeffizienten sich verändern, wenn einzelne Variablen weggelassen werden. Wir verfolgen jedoch noch eine weitere Strategie, um den Problemen der Multikollinearität gerecht zu werden, nämlich den Einsatz partialisierter Modelle. Durch dieses Vorgehen können wir die relative Bedeutung der Hauptvariablen im Vergleich mit anderen unabhängigen Variablen erfassen.

Für die Modelle 6 bis 11 in Tabelle 2 wurde der gemeinsame Einfluss unterschiedlicher unabhängiger Variablen sequentiell herausgefiltert. Dabei werden die unabhängigen Variablen orthogonalisiert, d. h. sie werden durch bivariate Regressionen und nachfolgende Residualisierung stochastisch unabhängig gemacht. Von den in der Hierarchie nachrangigen Variablen wird nur das unstandardisierte Residuum der bivariaten Regression mit der betreffenden höherrangigen Variablen in die Modellgleichung aufgenommen. Der Koeffizient einer untergeordneten Variablen beinhaltet also nur Informationen zu Effekten, die über den gemeinsamen Effekt der höherrangigen Variablen hinausgehen. Die Güte des multivariaten Modells wird von der Orthogonalisierung der unabhängigen Variablen nicht beeinflusst.

Die Partialisierung von Effekten der weniger wichtigen Variablen finder schrittweise statt. Die Variablen werden nach verschiedenen Gruppen geordnet. Jede Variable wird durch alle Variablen höherer Gruppen orthogonalisiert. Wir erläutern das Vorgehen anhand von Modell 6, für welches die folgende Regressionsgleichung gilt:

$$
\begin{aligned}
\mathrm{y}= & \mathrm{c}+\beta_{1 \mathrm{y}} \mathrm{x}_{1}+\beta_{2 \mathrm{y}} \mathrm{x}_{2}+\beta_{3 \mathrm{y}} \mathrm{x}_{3}+\beta_{(4-123) \mathrm{y}} \mathrm{x}_{4-123}+\beta_{(5-1234) \mathrm{y}} \mathrm{x}_{5-1234}+ \\
& \beta_{\left.\mathrm{c}_{\mathrm{i}}-12345\right) \mathrm{y}} \mathrm{x}_{\mathrm{c}_{\mathrm{i}}-12345}+\beta_{\left(\mathrm{t}-12345 \mathrm{c}_{\mathrm{i}}\right) \mathrm{y}} \mathrm{x}_{\mathrm{t}-12345 \mathrm{c}_{\mathrm{i}}}+\mathrm{e}
\end{aligned}
$$

In der Basisversion dieser partialisierten Regression werden aus den zwölf Variablen fünf verschiedene Gruppen für die Orthogonalisierung zusammengesetzt: Variablen $\mathrm{x}_{1}$ bis $\mathrm{x}_{3}$ sind Harmonisierungsvariablen (Gruppe 1), $\mathrm{x}_{4}$ steht für kommunikatives Potenzial (Gruppe 2), $x_{5}$ für Handel (Gruppe 3), $x_{c_{i}}$ repräsentiert die Kontrollvariablen $c_{i}$ (Gruppe 4) und $x_{t}$ ist die Zeitvariable (Gruppe 5).

Die hierarchische Ordnung aus Modell 6 impliziert bestimmte theoretische Erwartungen oder Annahmen im Hinblick auf die Rangordnung der Variablen. Erstens setzt sie voraus, dass die Konvergenzeffekte in folgender Reihenfolge schwächer werden: Für internationale Harmonisierung sind sie am stärksten, gefolgt von transnationaler Kom- 


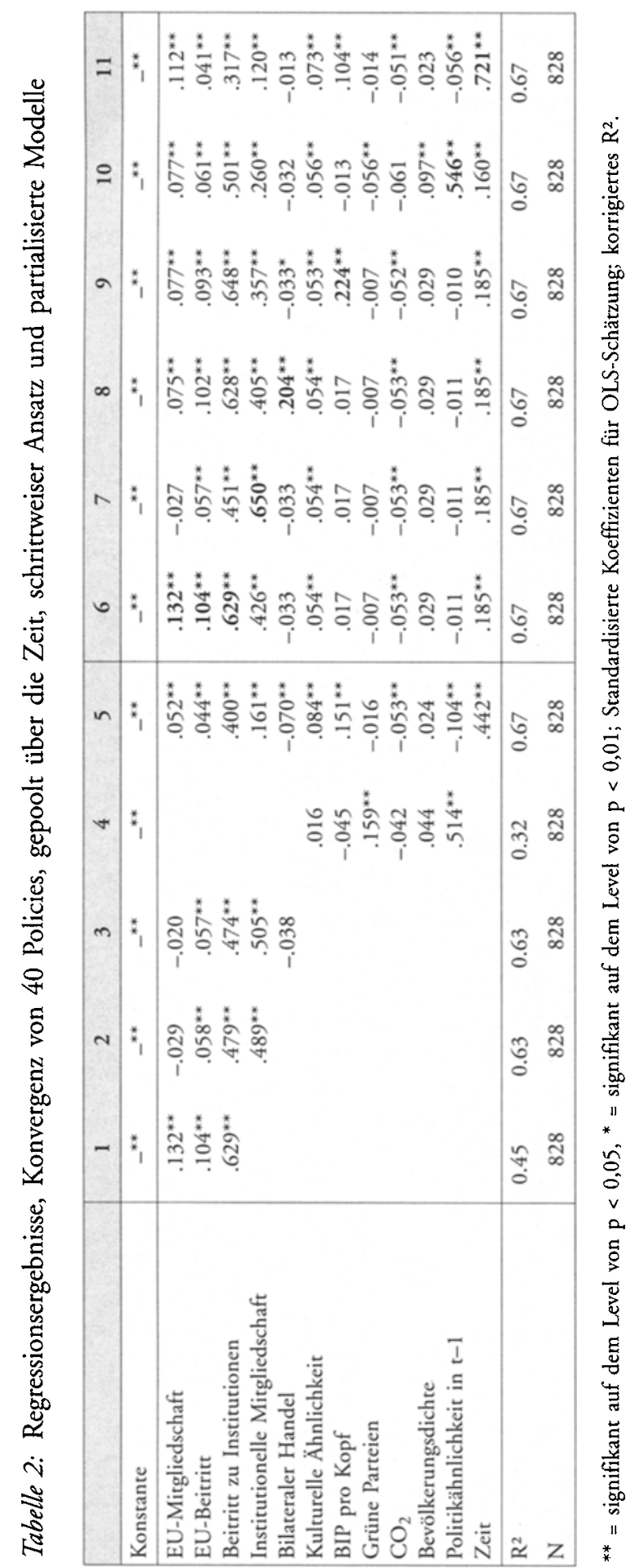




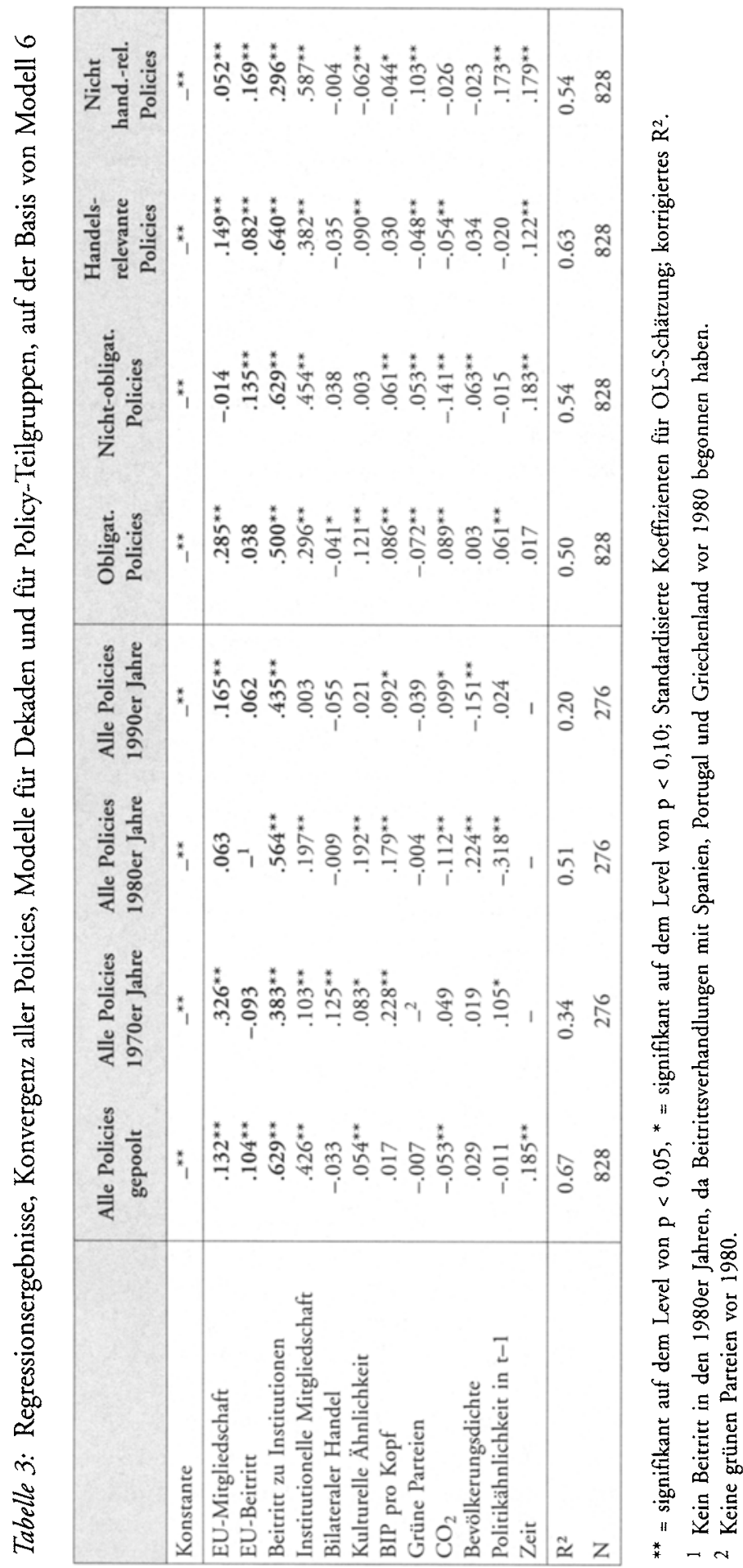


munikation und regulativem Wettbewerb. Bei den Kontrollvariablen liegt unser Interesse nicht so sehr auf ihrem Beitrag zur Erklärung von Konvergenz, sondern auf einem möglichen Effekt, der über die Hauptvariablen hinausgeht. Sie werden deshalb bezüglich der fünf Variablen, die sich auf die drei Haupttriebkräfte von Konvergenz beziehen, residualisiert. Die Zeitvariable nimmt den untersten Platz in der Hierarchie ein, da sie mit vielen Kovariaten verwandt ist und keine explizite theoretische Erwartung formuliert werden kann. Auf diese Weise geht nur der Zeiteffekt, der den Einfluss aller anderen Variablen übertrifft, in die Regression ein. Dieser Koeffizient sollte daher deutlich kleiner sein als im nicht-partialisierten Modell.

Modell 6 stellt nur eine von mehreren Möglichkeiten dar, die Variablen in eine Rangordnung zu bringen. Da wir über keine Theorien verfügen, auf deren Basis sich eine bestimmte Rangordnung überzeugend begründen ließe, sind auch andere Modelle denkbar. Um theoretische Beliebigkeit zu vermeiden, haben wir fünf weitere partialisierte Regressionsmodelle verwendet, mit je verschiedenen Variablenhierarchien. An der Spitze der Rankings in den Modellen 7 bis 11 stehen die Variablen für die transnationale Kommunikation, den Regulierungswettbewerb, sowie die wichtigsten Kontrollvariablen, nämlich der Grad der wirtschaftlichen Entwicklung, die schon bestehende Politikähnlichkeit und die Zeitvariable. Die Gleichung ist jeweils analog zu Modell 6. In den Tabellen 2 und 3 sind die Variablen, die an der Spitze der Hierarchie stehen, fett gedruckt.

Die gepoolten Modelle werden durch Querschnittsmodelle ergänzt (Tabelle 3). Dies erlaubt es, kausale Effekte in den Dekadenmodellen mit Effekten zu vergleichen, die über die gesamte Periode auftreten, und Veränderungen in der Bedeutung der kausalen Mechanismen über den gesamten Zeitraum zu untersuchen. Für die Modelle in Tabelle 3 benutzen wir das partialisierte Modell 6 als Grundlage. Der Grund dafür ist, dass die Harmonisierungsvariablen nicht nur den größten Effekt in Modell 6 aufzeigen, wo sie an der Spitze der Hierarchie stehen, sondern auch in allen anderen partialisierten Modellen über einen hohen Einfluss verfügen.

\subsection{Güte der Modelle}

Die in Tabelle 2 und 3 vorgestellten Modelle zeigen im Hinblick auf die erklärte Varianz zufriedenstellende Ergebnisse. Für das gepoolte Modell, das alle Variablen enthält (Modell 5 in Tabelle 2) ist die Varianzaufklärung mit einem korrgierten $R^{2}$ von .67 sehr hoch. Auch Modell 3, welches nur die wichrigsten unabhängigen Variablen enthält, zeigt ein vergleichbar hohes $\mathrm{R}^{2}$ (.63). Im Gegensatz dazu ist der Beitrag der Kontrollvariablen zur Varianzaufklärung eher gering (siehe Modell 4).

Die Güte der Modelle variiert aber stark, wenn man die drei Dekaden separar betrachtet. Tabelle 3 verweist auf ein hohes Erklärungspotenzial des Modells für Konvergenz zwischen 1980 und $1990\left(\mathrm{R}^{2}=.51\right)$, während die Werte für die 1970er $\left(\mathrm{R}^{2}=\right.$ .34) und 1990er Jahre $\left(\mathrm{R}^{2}=.20\right)$ deutlich niedriger liegen. Die Abweichung für die 1980er Jahre kann durch die Dynamik einige kausaler Mechanismen in dieser Periode erklärt werden. Der höhere Wert beim gepoolten Modell wird durch eine Parallelent- 
wicklung der abhängigen und einiger wichtiger unabhängiger Variablen über den gesamten Untersuchungszeitraum hervorgerufen.

\subsection{Regressionsergebnisse: Was erklärt die Konvergenz?}

In den folgenden Abschnitten werden die Ergebnisse der Regressionsanalyse für jede Erklärungsvariable einzeln vorgestellt. Wir gehen jeweils wie folgt vor: Zuerst analysieren wir die Effekte auf die umfassende abhängige Variable, die alle Policies enthält. Im zweiten Schritt vergleichen wir die Effekte in den verschiedenen Dekaden. Drittens differenzieren wir zwischen Policy-Teilgruppen. Gelegentlich wird auch auf die bivariaten Korrelationen verwiesen (vgl. Tabelle A4 im Anhang).

\section{Internationale Harmonisierung: Auswirkungen von EU-Mitgliedschaft}

Die Ergebnisse zeigen zunächst eine positive Korrelation zwischen EU-Mitgliedschaft und Politikkonvergenz und bestätigen somit unsere theoretischen Erwartungen. Obwohl signifikant, hat die EU-Mitgliedschaft allerdings nicht die größte Vorhersagekraft für Konvergenz. Während der standardisierte Koeffizient im partialisierten Modell 6 bei .132 liegt (wobei das Erklärungspotenzial aller anderen Variablen ja völlig auf die EU-Mitgliedschaft übertragen ist), fällt er in Modell 5, das alle unabhängigen Variablen enthält, auf .052 .

Die Korrelation zwischen EU-Mitgliedschaft und Konvergenz variiert im Zeitablauf (siehe Tabelle 3). Der Effekt der EU-Mitgliedschaft ist vor allem in den 1970er Jahren stark positiv (.326), in den 1980er Jahren hingegen sehr schwach (.062). In den $1990 \mathrm{er} \mathrm{Jahren} \mathrm{erfährt} \mathrm{sie} \mathrm{einen} \mathrm{erneuten} \mathrm{Anstieg} \mathrm{(.165).} \mathrm{Diese} \mathrm{Beobachtungen} \mathrm{passen}$ zur allgemeinen Entwicklung der EU-Umweltpolitik (Weale u. a. 2000). In den 1970er Jahren verursachte der Anstieg von Umweltaktivitäten der EU starke Konvergenzeffekte für die damaligen Mitglieder. In dieser Phase führte die EU bindende Regulierungen für neun von 19 obligatorischen Policies aus unserem Datensatz ein, während zwischen 1978 und 1990 nur vier obligatorische Regelungen getroffen wurden, die Policies aus unserem Datensatz betrafen. Abgesehen von Änderungen schon bestehender Gesetze ging von der EU-Ebene nur sehr geringer zusätzlicher Harmonisierungsdruck aus. Zwischen 1990 und 2000 stieg die Anzahl der neu eingeführten Regulierungen, die in unserem Datensatz vorkommen, auf sechs an. Dies erklärt den höheren Konvergenzeffekt für die 1990er Jahre.

Die Auswirkungen einer EU-Mitgliedschaft variieren auch stark im Hinblick auf die Konvergenz in den verschiedenen Teilgruppen. Im Allgemeinen sind die Effekte für obligatorische und handelsrelevante Policies sehr viel stärker als für solche, die keinen obligatorischen Charakter haben bzw. nicht handelsrelevant sind. Dieses Ergebnis lässt sich daraus erklären, dass die Umweltpolitik der EU stark vom Anliegen der Bildung eines gemeinsamen Marktes beeinflusst wurde (Holzinger 1994). Als Konsequenz konzentrieren sich EU-Aktivitäten vornehmlich auf handelsrelevante Policies und die Harmonisierung nationaler Produkt- und Prozessstandards, um Handelsschranken und Wettbewerbsverzerrungen zu vermeiden. 
Allgemein sind die Effekte der EU-Mitgliedschaft weniger groß als erwartet. Was sind die Gründe für dieses überraschende Ergebnis? Erstens waren bis 1995 nur zwölf von 24 Ländern Mitglieder der EU und in den zwei vorhergehenden Dekaden waren es sogar deutlich weniger als die Hälfte der Untersuchungsländer. Über den gesamten Zeitraum bilden EU-Mitgliedstaaten also nur einen kleinen Anteil der untersuchten Länder. Zweitens orientieren sich auch Nicht-Mitglieder in ihrer Politik an der EUGesetzgebung. ${ }^{4}$ Drittens zeigen unsere Daten signifikante Unterschiede zwischen den EU-Mitgliedstaaten bei der Rechtsumsetzung, da Umweltdirektiven normalerweise einen erheblichen Spielraum für die nationale Gesetzgebung offen lassen. Außerdem würde man im Fall von Minimumharmonisierung unter EU-Staaten ohnehin keine vollständige Konvergenz erwarten.

\section{Internationale Harmonisierung: EU-Beitritt}

Auch die Variable EU-Beitritt korreliert positiv mit der Konvergenz der Umweltpolitiken. Ähnlich wie bei der EU-Mitgliedschaft zeigt das partialisierte Modell 6 einen moderaten Effekt (.104), der in Modell 5, welches alle Variablen enthält, stark abnimmt.

Die Dekadenmodelle zeigen die Dynamik der EU-Beitrittseffekte. Für die 1970er Jahre ergibt sich ein negativer, wenn auch insignifikanter Effekt (-.093). Hierfür sind Griechenland, Portugal und Spanien verantwortlich, da sie den umweltpolitischen aquis nicht schon mit Beginn der Beitrittsgespräche 1978 übernommen haben, sondern erst allmählich nach ihrem offiziellen Beitritt. Für die 1990er Jahre ist der Koeffizient positiv (.062), wenn auch nicht sehr ausgeprägt. Dies könnte die Konsequenz der zwei Erweiterungen in den 1990er Jahren sein. Die erste Erweiterungsrunde begann in den frühen 1990 er Jahren und betraf Finnland, Österreich und Schweden. Die zweite Runde begann Mitte der 1990er Jahre und schloss drei osteuropäische Länder aus unserem Sample mit ein (Polen, Ungarn und die Slowakei). Eine Differenzierung zwischen den beiden Gruppen zeigt, dass für die erste Gruppe ein negativer Effekt zu sehen ist, während für die zweite Gruppe ein deutlich positiver Effekt beobachtet werden kann. Die EFTA-Länder hatten schon immer ähnliche Umweltpolitiken wie die EU-Länder; daher war der Konvergenzeffekt für diese Gruppe gering, während der Beitrittsprozess für die zweite Gruppe eine weit reichende Angleichung hervorgerufen hat.

\section{Internationale Harmonisierung: Beitritt zu Internationalen Institutionen}

Unsere theoretischen Erwartung, dass internationale Harmonisierung auch durch den Beitritt zu anderen internationalen Institutionen als der EU effektiv sein kann, wird durch unsere Ergebnisse eindrucksvoll bestätigt. Der Einfluss dieser Variable ist eindeutig stärker als der Einfluss der EU-Variablen (.629 im partialisierten Modell 6). Die Größe und Richtung des Effektes sind ziemlich stabil. Dies zeigt sich auch in Modell 5 (.400). Die Variable ist Indikator für das Ausmaß, mit dem nationale Regierungen an internationale Gesetzgebung gebunden sind. Dies scheint eine präzise Vorhersage von Konvergenz zu ermöglichen. Internationale Regulierungskooperationen zeigen offenbar Wirkungen, die deutlich über die EU-Effekte hinausgehen. In diesem Zusammenhang

4 Zum Beispiel führten Österreich und die Schweiz in den 1980er Jahren dieselben Grenzewerte für den Schwefelgehalt von Gasöl wie die EU ein. 
muss hervorgehoben werden, dass das Verhältnis zwischen dieser und der abhängigen Variablen kein tautologisches ist. Zwischen der Einführung einer internationalen Übereinkunft und ihrer nationalen Implementierung liegen viele Schritte und manche Hürden.

Im Zeitablauf gibt es eine gewisse Varianz bei den Effekten, wobei die Koeffizienten in den 1980er (.575) am stärksten und in den 1970er Jahren (.383) am schwächsten ausgeprägt sind. Der schwächere Effekt für die 1970er Jahre und der Höhepunkt in der zweiten Dekade kann damit erklärt werden, dass viele internationale Umweltregime, wie die Konvention zur langfristigen grenzübergreifenden Luftverschmutzung oder die Wiener Konvention zum Schutz der Ozonschicht, erst nach 1980 eingeführt wurden. Der leichte Abfall des positiven Effektes in den 1990er Jahren könnte durch das hohe $\mathrm{Maß}$ an institutioneller Integration hervorgerufen sein, die in der vorhergehenden Periode bereits erreicht war.

Ein deutlich positiver Effekt kann sowohl für die Konvergenz aller Policies als auch für alle Policy-Teilgruppen konstatiert werden. Die Erklärungskraft des Beitritts zu internationalen Institutionen ist für obligatorische, nicht-obligatorische und handelsrelevante Policies auf einem ähnlich hohen Niveau.

Insgesamt zeigt sich, dass die Variablen, die sich auf die internationale Harmonisierung beziehen, das Auftreten von Politikkonvergenz von 1970 bis 2000 sehr gut erklären können. Der Harmonisierungseffekt ist dominant im Regressionsmodell 5, das alle unabhängigen Variablen enthält. Aus diesem Grund wurde das partialisierte Modell 6, in dem alle Variablen den Harmonisierungsvariablen untergeordnet sind, als Grundlage für die in Tabelle 3 vorgestellten Modelle ausgewählt. Deshalb könnten die Harmonisierungseffekte im Hinblick auf ihre absolute Größe in diesen Modellen etwas uiberbewertet sein, nicht jedoch im Hinblick auf ihre relative Größe.

\section{Transnationale Kommunikation: Institutionelle Mitgliedschaft}

Unsere Regressionsergebnisse zeigen nicht nur starke Konvergenzeffekte als Ergebnis internationaler Harmonisierung, sondern sie unterstützen auch die Erwartung einer ähnlichen Auswirkung von Kommunikation und Informationsaustausch in transnationalen Netzwerken. Die Konvergenzeffekte gemeinsamer Mitgliedschaft in internationalen Organisationen (.489 in Modell 2) sind von ihrer Stärke her mit denen der internationalen Harmonisierung vergleichbar. Das maximale Erklärungspotenzial der Variable wird im partialisierten Modell 7 deutlich, in dem alle anderen Koeffizienten nur als über die Mitgliedschaft in internationalen Institutionen hinausreichende Effekte interpretiert werden können. Modell 6 zeigt gleichzeitig, dass der starke positive Einfluss sogar dann bestehen bleibt (.426), wenn nur solche Effekte gemeinsamer institutioneller Mitgliedschaft berücksichtigt werden, die über internationale Harmonisierung hinausgehen. Gemeinsame Mitgliedschaft in Institutionen mit hohem kommunikativen Potenzial ist also einer der einflussreichsten Erklärungsfaktoren für transnationale Politikkonvergenz.

Der Einfluss gemeinsamer Mitgliedschaft variiert jedoch über den Zeitablauf. Für die 1970er (.103) und 1980er Jahre (.197) trägt die Variable positiv zur Erklärung der Konvergenz aller Policies bei, während der Effekt in den 1990er Jahren vollständig verschwindet. Dieser auffällige Wandel in der Auswirkung transnationaler Kommunikation lässt sich dadurch erklären, dass Länderpaare mit starker kommunikativer Ver- 
flechtung aufgrund von Saturierungseffekten in den 1990er Jahren nur in geringerem Maß konvergieren (können) als in den vorhergehenden Perioden. Ein genauerer Blick auf die schon vor 1990 erreichte Politikähnlichkeit zeigt, dass die Kommunikation die Konvergenz in diesen Ländern in den 1990er Jahren nicht beeinflusst hat.

Die generelle Aussage über die starke Erklärungskraft transnationaler Kommunikation gilt auch für verschiedene Policy-Teilgruppen. Während die Effekte für alle Gruppen stark sind, sind sie für Policies, die nicht obligatorisch oder nicht handelsrelevant sind, besonders ausgeprägt. Dies kann auf die Tatsache zurückgeführt werden, dass es für die Konvergenz von obligatorischen und handelsrelevanten Policies keinen signifikanten Kommunikationseffekt gibt, der den der Harmonisierungsvariablen übertrifft. Der trotz allem signifikante Effekt bei diesen Policies muss keinen Widerspruch darstellen. So erscheint es etwa durchaus plausibel, dass auf EU-Ebene verabschiedete Policies breite internationale Beachtung finden und daher auch in Ländern übernommen werden, die (noch) nicht EU-Mitglieder sind. Bemerkenswert ist in diesem Zusammenhang, dass bis 1990 nur 30 Prozent aller Länderpaare gemeinsam EU-Mitglieder waren.

\section{Regulativer Wettbewerb: Bilateraler Handel}

Im Gegensatz zu den bisher analysierten Variablen unterstützen unsere Ergebnisse die Hypothese, dass ein hoher Grad an wirtschaftlicher Verflechtung zu einer steigenden Ähnlichkeit ihrer nationalen Politiken führt, nur bedingt. Nur in Modell 8 , in dem alle Variablen bilateralem Handel untergeordnet sind, ist die Korrelation zwischen bilateraler Handelsoffenheit und Konvergenz signifikant und eindeutig positiv (.204). In den übrigen Modellen verschwindet der Handelseffekt, und der Koeffizient ist sogar leicht negativ. Diese Ergebnisse stimmen mit denen anderer Studien überein, die keinen Anhaltspunkt für einen solchen Handelseffekt auf Konvergenz oder Diffusion finden (vgl. Drezner 2001; Simmons/Elkins 2004).

Für dieses Ergebnis könnte eine Rolle spielen, dass die Variablen für wirtschaftliche und institutionelle Integration in einem gewissen Umfang durch Endogenität gekennzeichnet sind. Dies gilt insbesondere für die EU. Institutionelle Integration führt zu größeren Handelsbewegungen und die Existenz wirtschaftlicher Integration bildet für sich genommen einen Anreiz, in politischen Institutionen auf internationaler Ebene zu kooperieren. Offenbar ist der Faktor der internationalen Kooperation stärker als die Regulierungskonkurrenz. Während es in Modell 6 keinerlei Handelseffekt über Harmonisierung und Kommunikation hinaus gibt (-.033), sind die Koeffizienten für die institutionellen Variablen auch in Modell 8 stabil, in dem sie der Handelsoffenheit untergeordnet sind. Dies spricht für die Vermutung, dass der Einfluss von Wettbewerb weniger wichtig ist und von der institutionellen Integration übertroffen wird. Es erscheint plausibel, dass potentielle Wettbewerbseffekte durch die beteiligten Länder antizipiert und durch internationale Harmonisierung reduziert werden.

Unterstützt wird diese Interpretation, wenn man die Effekte der bilateralen Handelsoffenheit über den Zeitablauf beobachtet. Handelseffekte, die über Harmonisierung hinaus gehen, sind für die 1970er Jahre, als die internationale Kooperation in der Umweltpolitik an ihrem Anfang stand, ausgeprägt und signifikant positiv (.125). Über den Zeitablauf verschwindet dieser Effekt vollständig und ist für die $1990 \mathrm{er}$ Jahre sogar ne- 
gativ. Daher scheint gegenseitige Handelsabhängigkeit für die Konvergenz in den letzten Jahren eine untergeordnete Rolle zu spielen.

Auch bei den untersuchten Policy-Teilgruppen können wir keinen wichtigen Effekt bilateralen Handels auf Konvergenz ausmachen. Besonders interessant ist, dass der Koeffizient für handelsrelevante Policies (für die Effekte des Regulierungswettbewerbs stark ausgeprägt sein sollten) sogar negativ ist. Dies unterstützt erneut die Interpretation, dass Handelseffekte durch internationale Harmonisierung antizipiert werden.

\section{Kontrollvariablen}

Im Hinblick auf das Erklärungspotenzial der anderen Variablen sind unsere Befunde nicht eindeutig. Erstens beobachten wir sowohl in Modell 5, als auch in allen partialisierten Modellen, für kulturelle Ähnlichkeit einen leichten positiven Effekt auf transnationale Konvergenz (.084). Die Effekte variieren im Zeitablauf. Während sie in den 1980er Jahren am stärksten ausgeprägt sind, verschwinden sie in den 1990er Jahren fast völlig. Dieser Trend wird durch die bivariate Regression bestätigt (Tabelle A4). Die starken Effekte kultureller Ähnlichkeit in den 1970er und 1980er Jahren zeigen sich in einer Periode, die durch eine starke Expansion von Umweltpolitiken auf der europäischen und der nationalen Ebene gekennzeichnet ist. Es ist gut vorstellbar, dass diese dynamische Entwicklung Länder inspiriert hat, innovative Policies von kulturell ähnlichen und Nachbarländern zu kopieren und zu adaptieren. Diese Interpretation wird durch die Tatsache gestützt, dass kulturelle Ähnlichkeit für die 1990er Jahre, die generell als eine Stagnationsphase der Umweltpolitik angesehen werden, kein relevanter Faktor für Konvergenz ist. Die Entwicklung geht einher mit der zunehmenden Bedeutung internationaler Institutionen und scheint von diesem Mechanismus ersetzt zu werden, auch weil während der 1990er Jahre vor allem periphere Länder der Kerngruppe europäischer Staaten näher kamen. Bei den Teilgruppen sind die Effekte kultureller Ähnlichkeit für obligatorische Policies überraschend stark. Da dies nicht auf EU-Effekte zurückgeführt werden kann (dies ist aufgrund der Partialisierung ausgeschlossen), könnte die Erklärung erneut in der Emulation von EU-Politiken durch kulturell ähnliche Länder liegen, die noch keine Mitglieder der EU sind.

Für die zweite Kontrollvariable bestätigt sich die Hypothese: Ein hohes und gleich bleibendes Einkommensniveau eines Länderpaares führt zu Konvergenz. Wir beobachten positive Einkommenseffekte im Modell 5 (.151). Doch nehmen die Koeffizienten stark $a b$, wenn es um Effekte geht, die über die der institutionellen Variablen hinaus gehen. Vergleicht man die partialisierten Modelle 6, 7 und 9, finden wir ein ähnliches Szenario wie beim Regulierungswettbewerb vor: Während die institutionellen Effekte in Modell 9, das durch das Einkommensniveau orthogonalisiert ist, stabil sind, verschwinden die Einkommenseffekte in den Modellen 6 und 7 (in denen institutionelle Harmonisierung und Kommunikation an der Spitze der Rangordnung stehen). Ähnlich wie beim Regulierungswettbewerb kann dies als das Fehlen einer unabhängigen Auswirkung wirtschaftlicher Entwicklung auf Konvergenz interpretiert werden. Eine weitere Parallele zu den Handelseffekten ist die Existenz eines positiven Einflusses auf Konvergenz, der unabhängig von institutionellen Effekten für die 1970er und 1980er Jahre besteht, in den 1990 er Jahren aber verschwindet. Diese Entwicklung kann darauf zu- 
rückgeführt werden, dass reiche Länder schon sehr ähnlich waren und nicht mehr weiter konvergierten, während arme Länder gerade in den 1990er Jahren aufrückten.

Drittens geben unsere Ergebnisse wenig Anhaltspunkte für einen Einfluss politischer Nachfrage auf transnationale Politikkonvergenz. Ein signifikanter positiver Effekt zeigt sich nur in Modell 4 (.151), in dem alle relevanten Hauptvariablen ausgeschlossen sind. Dies trifft auch für die Dekadenmodelle zu. Nur für die Teilgruppen der nichtobligatorischen und nicht-handelsrelevanten Policies findet sich ein Effekt, der über den Einfluss der Hauptvariablen hinausgeht. Das heißt, wir finden Effekte für Policies, die mit internationaler Interdependenz und Harmonisierung kaum verbunden sind und allein auf der Basis nationaler Erwägungen beschlossen werden. Das bestätigt die Vermutung, dass die politische Nachfrage nationale Politikpräferenzen beeinflusst. Im Allgemeinen scheint diese Variable kein unabhängiger Mechanismus zu sein, der die transnationale Konvergenz nachhaltig beeinflusst hätte. Der Mangel an Effekten in den frühen Dekaden ist jedoch kaum verwunderlich, da es bis Mitte der 1980er Jahre nur in wenigen der untersuchten Länder grüne Parteien gab.

Viertens ergibt sich ein ähnliches Ergebnis für die zwei Variablen, die den umweltpolitischen Problemdruck messen $\left(\mathrm{CO}_{2}\right.$-Emmissionen und Bevölkerungsdichte), Fast alle multivariaten Modelle zeigen nur geringe oder keine Erklärungskraft. Allerdings gibt es zu dieser generellen Aussage einige Ausnahmen: Einerseits gibt es für $\mathrm{CO}_{2}$-Emissionen und für die Untergruppe der obligatorischen Policies signifikante positive Effekte in den 1990er Jahren. Beide Effekte können kausal mit dem Aufholen der osteuropäischen Länder verknüpft werden, in denen der umweltpolitische Problemdruck sehr hoch gewesen ist, vor 1990 aber eben ohne Konsequenzen für die Politik. Andererseits ist der Effekt der Bevölkerungsdichte für die Teilgruppe der nicht-obligatorischen Policies in den 1980er Jahren besonders ausgeprägt. Dies ist darauf zurückzuführen, dass es in der Gruppe der nicht-obligatorischen Policies einen großen Anteil an Wasserpolitiken gibt, die in Gebieten mit hoher Bevölkerungsdichte von besonderer Wichtigkeit sind. Die relativ starken Effekte in den 1980er Jahren können damit erkärt werden, dass es in dieser Zeit in den industrialisierten Ländern eine starke Entwicklung von umweltpolitischen Aktivitäten gab.

Die Ergebnisse bezüglich des Einkommensniveaus, der politischen Nachfrage und des umweltpolitischen Problemdrucks sind weniger überraschend, wenn wir uns daran erinnern, dass wir ihre Auswirkung auf den Grad der Konvergenz analysieren. Für alle drei Faktoren würden wir eher einen Effekt auf die Richtung von Konvergenz erwarten, nämlich dass sie sich in Richtung strengerer umweltpolitischer Auflagen bewegen.

Fünftens zeigen Modell 4 und das partialisierte Modell 10 für die schon bestehende Politikähnlichkeit eine sehr hohe positive Korrelation mit Konvergenz (.514; .546). Das gleiche gilt für die bivariate Korrelation (Tabelle A4). Dies bedeutet, dass Länderpaare, zwischen deren Umweltpolitiken schon ein hoher Grad an Ähnlichkeit besteht, in der Folge stärker konvergieren als Paare mit weniger Ähnlichkeit. Im allgemeinen multivariaten Modell 5 gibt es aber keinerlei unabhängigen Effekt, der über den Einfluss der internationalen Triebkräfte von Konvergenz hinausgeht. Die Analyse der Dekaden hilft, dieses widersprüchliche Bild zu deuten. Wir finden signifikant positive Effekte für die 1970er Jahre (.105), einen negativen Effekt für die 1980er Jahre (-.318) und einen unwesentlichen Effekt für die 1990er Jahre. Die 1970er Jahre waren eine Zeit, in der 
sich unweltpolitische Pionierländer aneinander angeglichen haben. In den 1980er Jahren hatte die Konvergenz zwischen einigen Ländern schon ein hohes Niveau erreicht, so dass wenig Potenzial für Konvergenz in nachfolgenden Perioden blieb. Diese Entwicklung kann als Folge von Saturierungseffekten interpretiert werden: Gleichzeitig zeigten Länder, die vorher weniger ähnlich waren (da sie über keine oder wenig bestehende Policies verfügten) in den $1990 \mathrm{er}$ Jahren wesentlich höhere Konvergenzraten. Daher wird Konvergenz hier besser durch vorherige Nicht-Ähnlichkeit erklärt. Dieser Wandel kann den fundamentalen Veränderungen in den Umweltpolitiken der osteuropäischen Länder und Mexiko zugeschrieben werden. Der negative Effekt oder das Fehlen von Korrelation in den späteren Perioden weist also auf einen Aufholprozess der langsameren Länder hin.

Schließlich hat auch die Zeit einen Einfluss auf Politikkonvergenz. Wir haben keine spezifischen theoretischen Erwartungen im Hinblick auf die Effekte dieser Variable. Sie repräsentiert die Dynamik dieses relativ neuen Politikbereiches und schließt Aspekte mit ein, die die Entwicklung der Umweltpolitik im Allgemeinen antreiben, aber in dieser Analyse nicht auftauchen. Beispiele sind technischer Fortschritt, Kostenreduzierung von Umweltschutzmaßnahmen, und exogene Schocks wie saurer Regen, die nukleare Katastrophe von Tschernobyl oder der Treibhauseffekt. Im gepoolten Modell korreliert Zeit stark mit Konvergenz. Dies gilt für das allgemeine Modell 5 (.442) und für alle Modelle mit partialisierten Variablen, in denen der Zeiteffekt über alle anderen Variablen hinaus immer signifikant positiv ist. Modell 11 zeigt, dass der Zeiteffekt sehr stark ist, wenn alle anderen Faktoren partialisiert sind (.721). Doch selbst in diesem Modell haben Harmonisierung, transnationale Kommunikation und Einkommen noch eigenständige Erklärungskraft.

\section{Fazit}

Die Analyse der Konvergenz von Umweltpolitiken erlaubt einige generelle Schlussfolgerungen. Erstens zeigen unsere Ergebnisse, dass die Ähnlichkeit von Umweltpolitiken in den untersuchten Ländern in der beobachteten Periode (1970 bis 2000) stark angestiegen ist. Wie erwartet variiert der Grad der Konvergenz über die verschiedenen Typen und Dimensionen von Policies. Er ist bei obligatorischen und handelsrelevanten Policies ausgeprägter als bei nicht-obligatorischen und nicht-handelsrelevanten Policies und er zeigt sich am stärksten für die Dimension der Politikpräsenz und am schwächsten für die Dimension des konkreten Regulierungsniveaus.

Zweitens lassen sich diese Entwicklungen besonders durch die Effekte internationaler Harmonisierung und transnationaler Kommunikation erklären. Die internationale Harmonisierung, die durch drei Variablen repräsentiert wird (EU-Mitgliedschaft, EUBeitritt, und Beitritt zu anderen internationalen Institutionen als der EU), trägt am meisten zur Erklärung von Konvergenz bei. Die Erklärungskraft der EU-Variablen ist geringer ausgeprägt als dies für den Beitritts zu internationalen Institutionen der Fall ist. Die Effekte transnationaler Kommunikation auf umweltpolitische Konvergenz sind fast genauso groß wie die der internationalen Harmonisierung. Die Kommunikationseffekte sind bei nicht-obligatorischen und nicht-handelsrelevanten Policies besonders 
stark ausgeprägt. Besteht schon eine harmonisierte Politik auf internationaler Ebene, gibt es wenig Raum dafür, dass der Einfluss transnationaler Kommunikation weitere Konvergenz bedingt. Da handelsrelevante Policies öfter Gegenstand internationaler Harmonisierung sind als nicht-handelsrelevante Policies, gibt es in dieser Gruppe eine hohe Überschneidung mit obligatorischen Policies.

Drittens hat der Regulierungswettbewerb verglichen mit den institutionellen Variablen wenig Einfluss auf die transnationale Politikkonvergenz. Wir finden keinerlei Handelseffekte, die über die Effekte von Harmonisierung oder Kommunikation hinausgehen. Das globale Regieren scheint also stärkere Auswirkungen auf die Politikkonvergenz zu haben als die wirtschaftliche Globalisierung.

Viertens scheint die Erklärungskraft der Kontrollvariablen eher gering zu sein. Während für Einkommen und kulturelle Ähnlichkeit einige Effekte beobachtet werden können, sind politische Nachfrage und umweltpolitischer Problemdruck nur von geringer Relevanz. Wichtig ist hier, dass für die schon bestehende Politikähnlichkeit starke Effekte beobachtet werden können. Länder, die schon über einen hohen Grad an Ähnlichkeit verfügten, konvergierten in nachfolgenden Perioden stärker. In späteren Perioden beobachten wir einen Aufholprozess der Nachzüglerstaaten.

\section{Literatur}

Barrios, Salvador/Görg, Holger/Strobl, Eric, 2003: Multinational Enterprises and New Trade Theory: Evidence for the Convergence Hypothesis, in: Open Economies Review 14, 397-418.

Bennett, Colin, 1991: What Is Policy Convergence and What Causes It?, in: British Journal of Political Science 21, 215-233.

Boyer, Robert, 1996: The Convergence Hypothesis Revisited: Globalization but still the Century of Nations?, in: Berger, Suzanne/Dore, Robert (Hrsg.), National Diversity and Global Capitalism. Ithaca, NY, 29-59.

Cowles, Maria Green/Caporaso, James A./Risse, Thomas (Hrsg.), 2001: Transforming Europe. Europeanization and Domestic Change. Ithaca, N. Y.

DiMaggio, Paul J./Powell, Walter W., 1991: The Iron Cage Revisited. Institutionalised Isomorphism and Collective Rationality in Organizational Fields, in: Powell, Walter W./DiMaggio, Paul J. (Hrsg.), The New Institutionalism in Organizational Analysis. Chicago, 63-82.

Dimitrova, Antoaneta/Steunenberg, Bernard, 2000: The Search for Convergence of National Policies in the European Union. An Impossible Quest?, in: European Union Politics 1, 201-226.

Dolowitz, David P./Marsh, David, 2000: Learning from Abroad: The Role of Policy Transfer in Contemporary Policy Making, in: Governance 13, 5-24.

Drezner, Daniel W., 2001: Globalization and Policy Convergence, in: International Studies Review 3, 53-78.

Garrett, Geoffrey, 1999: Partisan Politics in the Global Economy. New York.

Gilardi, Fabrizio, 2005: The International Foundations of Regulatory Capitalism: The Diffusion of Independent Regulatory Agencies in Western Europe, in: The Annals of the American Academy of Political and Social Science 598, 84-101.

Guillén, Mauro F, 2001: Is Globalization Civilizing, Destructive of Feeble? A Critique of Five Key Debates in the Social Science Literature, in: Annual Review of Sociology 27, 235-260.

Heichel, Stephan/Pape, Jessica/Sommerer, Thomas, 2005: Is There Convergence in Convergence Research? An Overview of Empirical Studies on Policy Convergence, in: Journal of European Public Policy 12, 817-840.

Héritier, Adrienne/Kerwer, Dieter/Knill, Christoph/Lehmkuhl, Dirk/Teutsch, Michael, 2001: Differential Europe. New Opportunities and Constraints for National Policy-Making. Lanham. 
Hoberg, George, 2001: Globalization and Policy Convergence: Symposium Overview, in: Journal of Comparative Policy Analysis: Research and Practice 3, 127-132.

Holzinger, Katharina, 1994: Politik des kleinsten gemeinsamen Nenners? Umweltpolitische Entscheidungsprozesse in der EG. Berlin.

Holzinger, Katharina/Knill, Christoph, 2005: Causes and Conditions of Cross-national Policy Convergence, in: Journal of European Public Policy 12, 775-796.

Holzinger, Katharina, 2003: Common Goods, Matrix Games, and Institutional Solutions, in: European Journal of International Relations 9, 173-212.

Holzinger, Katharina, 2006: Methodological Pitfalls of Convergence Analysis, in: European Union Politics 7, 271-287.

Kern, Kristine, 2000: Die Diffusion von Politikinnovationen. Umweltpolitische Innovationen im Mehrebenensystem der USA. Opladen.

Kern, Kristine/Jörgens, Helge/Jänicke, Martin, 2000: Die Diffusion umweltpolitischer Innovationen. Ein Beitrag zur Globalisierung von Umweltpolitik, in: Zeitschrift für Umweltpolitik und Umweltrecht 23, 507-546.

Knill, Christoph, 2001: The Europeanisation of National Administrations. Cambridge.

Martin, Lisa/Simmons, Beth, 1998: Theories and Empirical Studies of International Institutions, in: International Organization 52, 729-757.

Panayotou, Theodore, 1993: Empirical Tests and Policy Analysis of Environmental Degradation at Different Stages of Economic Development, in: ILO Technology and Employment Programme Working Paper, WP 238. Geneva.

Scharpf, Fritz W., 1997: Introduction. The Problem-solving Capacity of Multi-level Governance, in: Journal of European Public Policy 4, 520-538.

Schimmelfennig, Frank/Sedelmeier, Ulrich, 2004: Governance by Conditionality: EU Rule Transfer to the Candidate Countries of Central and Eastern Europe, in: Journal of European Public Policy 11, 661-679.

Simmons, Beth A./Elkins, Zachary, 2004: The Globalization of Liberalization. Policy Diffusion in the International Political Economy, in: American Political Science Review 98, 171-189.

Stern, David I./Common, Mick S., 2001: Is there an Environmental Kuznets Curve for Sulfur?, in: Journal of Environmental Economics and Management 41, 162-178.

Strang, David/Meyer, John W., 1993: Institutional Conditions for Diffusion, in: Theory and Society $22,487-511$.

Vogel, David, 1995: Trading Up. Consumer and Environmental Regulation in the Global Economy. Cambridge. 
Annex

Tabelle A1: Liste der Policies

\begin{tabular}{|c|c|c|}
\hline & $\begin{array}{c}\text { Handelsbezogene } \\
\text { Policies }\end{array}$ & $\begin{array}{l}\text { Obligatorische Policies } \\
\text { (verbindlich seit ...) }\end{array}$ \\
\hline Schwefelgehalt von Gasöl & • & 1975 \\
\hline Bleigehalt von Kraftstoff & • & 1978 \\
\hline PKW NOx-Emissionen & $\bullet$ & 1977 \\
\hline PKW CO-Emissionen & • & 1970 \\
\hline PKW HC-Emissionen & $\bullet$ & 1970 \\
\hline $\mathrm{SO}_{2}$-Emissionen von Großfeuerungsanlagen & $\bullet$ & 1988 \\
\hline $\mathrm{NO}_{\mathrm{x}}$-Emissionen von Großfeuerungsanlagen & $\bullet$ & 1988 \\
\hline Staubemissionen von Großfeuerungsanlagen & $\bullet$ & 1988 \\
\hline Kolibakterien in Badegewässern & & 1976 \\
\hline Gefährliche Substanzen in Reinigungsmitteln & • & 1973 \\
\hline Effiziente Wassernutzung in der Industrie & • & \\
\hline Bleigehalt in industriellen Abwässern & • & \\
\hline Zinkgehalt in industriellen Abwässern & • & \\
\hline Kupfergehalt in industriellen Abwässern & $\cdot$ & \\
\hline Chromgehalt in industriellen Abwässern & • & \\
\hline Biochemischer Sauerstoffbedarf in industriellen Abwässern & $\cdot$ & \\
\hline \multicolumn{3}{|l|}{ Bodenschutz } \\
\hline \multicolumn{3}{|l|}{ Altlastensanierung } \\
\hline Abfallziel Wiederverwertung & & 1994 \\
\hline Abfallziel Deponierung & & 1994 \\
\hline \multicolumn{3}{|l|}{ Wiederverwertungsziel Glas } \\
\hline \multicolumn{3}{|l|}{ Wiederverwertungsziel Papier } \\
\hline Förderung von wiederverwendbaren Getränkeverpackungen & • & \\
\hline Freiwilliges Flaschenpfand & • & \\
\hline Lärmschutzstandard bei LKW & • & 1970 \\
\hline \multicolumn{3}{|l|}{ Autobahn Lärmbelastung } \\
\hline Lärmbelastung am Arbeitsplatz & • & 1977 \\
\hline Stromerzeugung aus erneuerbaren Energien & • & \\
\hline Bauschutt-Recycling & • & \\
\hline Energieeffizienz bei Kühlschränken & • & 1992 \\
\hline \multicolumn{3}{|l|}{ Stromsteuer für private Haushalte } \\
\hline Industrielle Abgabe auf Schweröle & • & 1992 \\
\hline $\mathrm{CO}_{2}$-Emissionen durch Schwerindustrie & $\cdot$ & \\
\hline \multicolumn{3}{|l|}{ Forstschutz } \\
\hline Oko-Audit & • & 1993 \\
\hline Umweltverträglichkeitsprüfung & & 1985 \\
\hline Oko-Label & • & 1992 \\
\hline \multicolumn{3}{|l|}{ Vorsorgeprinzip } \\
\hline Nachhaltigkeitsprinzip: Gesetzesbezug & & \\
\hline Umwelt/Nachhaltigkeits-Entwicklungsplan & • & \\
\hline
\end{tabular}




\section{Tabelle A2: Berechnung der Konvergenz}

\begin{tabular}{|c|c|c|}
\hline $\begin{array}{l}\text { Ähnlichkeitswerte für die } \\
\text { Dimensionen } \\
\text { Politikpräsenz und } \\
\text { Instrumente }\end{array}$ & (1) & $\begin{array}{l}S P_{x, \underline{A B}}=1 \Leftrightarrow P_{x, A}=P_{x, B}>0 \\
S P_{x, \underline{A B}}=0 \Leftrightarrow P_{x, A} \neq P_{x, B}>0 \\
S I_{y, \underline{A B}}=1 \Leftrightarrow I_{y, A}=I_{y, B}>0 \\
S I_{y, \underline{A B}}=0 \Leftrightarrow I_{y, A} \neq I_{y, B}>0 \\
S P_{x} \text { bezieht sich auf die Ähnlichkeit der Policy } x, S I_{y} \text { auf die Ähnlichkeit } \\
\text { des Instruments } y, P_{x} \text { auf die Politikpräsenz } x, I_{y} \text { auf das Instrument } y \text { und } \\
\underline{A B} \text { auf die Dyade der Länder A und B. }\end{array}$ \\
\hline $\begin{array}{l}\text { Ähnlichkeitswerte für die } \\
\text { Dimension } \\
\text { Regulierungsniveau }\end{array}$ & (3) & $\begin{array}{l}S S_{Z, \underline{A B}}=1-\frac{\left|S_{z, A}-S_{z, B}\right|}{S_{z, 90}-S_{z, 10}} \\
\text { wobei } S S_{Z} \text { die Ähnlichkeit des Regulierungsniveaus } \mathrm{z} \text { ist, } S_{z, A} \text { und } S_{z, B} \\
\text { das Niveau der Regulierung } z \text { für die Länder A und B, und } S_{z, 90} \text { und } S_{z, 10} \\
\text { das } 90 \text {-Prozent- und das } 10 \text {-Prozent-Quantil der empirischen Verteilung } \\
\text { der Werte } z \text { in der Stichprobe sind. }\end{array}$ \\
\hline $\begin{array}{l}\text { Berechnung der } \\
\text { Konvergenzwerte }\end{array}$ & (4) & 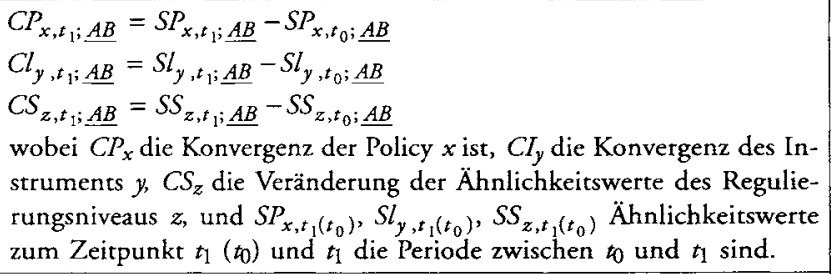 \\
\hline $\begin{array}{l}\text { Aggregation der } \\
\text { Konvergenzwerte }\end{array}$ & (5) & 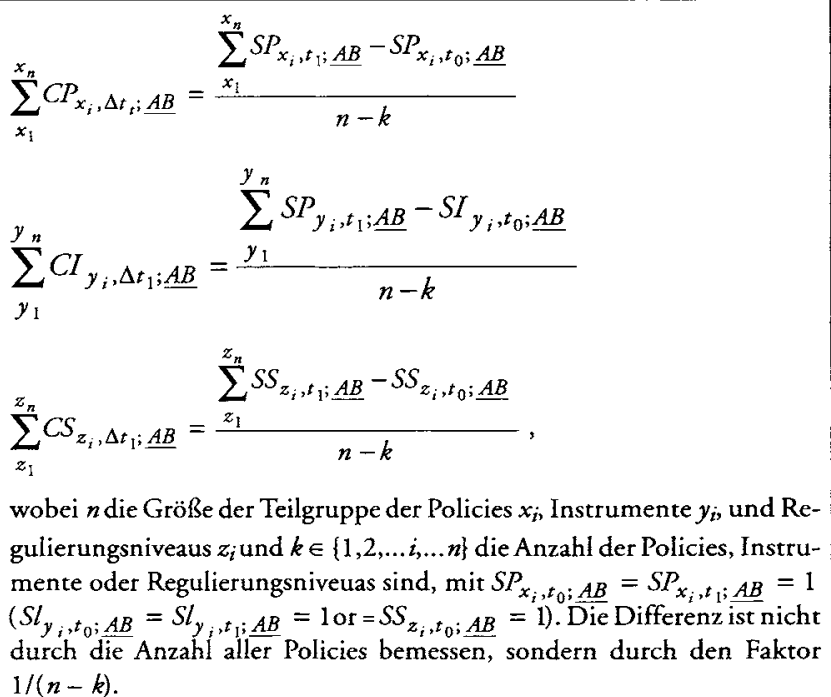 \\
\hline
\end{tabular}


Tabelle A3: Indikatoren und Indexkonstruktion

\begin{tabular}{|c|c|c|c|c|c|}
\hline \multirow[t]{2}{*}{ Indikator } & \multirow[t]{2}{*}{ Kategorien } & \multirow[t]{2}{*}{ Wert } & \multirow[t]{2}{*}{ Skala } & \multicolumn{2}{|c|}{ Aggregation } \\
\hline & & & & $\begin{array}{l}\text { Index // } \\
\text { normalisiert }\end{array}$ & $\begin{array}{c}\text { Cronbachs } \\
\alpha\end{array}$ \\
\hline \multicolumn{6}{|c|}{ Institutionelle Verflechtung } \\
\hline $\begin{array}{l}\text { Institutionelle } \\
\text { Mitgliedschaft }\end{array}$ & $\begin{array}{l}\text { Mitgliedschaft in } 35 \text { inter- } \\
\text { nationalen Institutionen }\end{array}$ & $(0 / 1)$ & $0-35$ & & \\
\hline \multicolumn{2}{|l|}{ Aktivitätsumfang } & & & $0-9 / / 0-1$ & 0,8 \\
\hline $\begin{array}{l}\text { Politikbereiche } \\
(1-14)\end{array}$ & $\begin{array}{l}\text { Fast alle Bereiche }(>10) \\
\text { Viele Bereiche }(5-1) \\
\text { Wenige Bereiche }(<5)\end{array}$ & $\begin{array}{l}3 \\
2 \\
1\end{array}$ & $0-3$ & & \\
\hline $\begin{array}{l}\text { Zahl der } \\
\text { Umweltthemen }\end{array}$ & $\begin{array}{l}\text { Alle Umweltthemen } \\
\text { Mehrere Umweltthemen } \\
\text { Nur ein Umweltthema }\end{array}$ & $\begin{array}{l}3 \\
2 \\
1\end{array}$ & $0-3$ & & \\
\hline \multirow[t]{3}{*}{$\begin{array}{l}\text { Bedeutung der } \\
\text { Anliegen }\end{array}$} & $\begin{array}{l}\text { Umweltpolitik ist wichtig, } \\
\text { aber nicht das einzige } \\
\text { Anliegen }\end{array}$ & 3 & $0-3$ & & \\
\hline & $\begin{array}{l}\text { Umweltpolitik spielt eine } \\
\text { dominante Rolle }\end{array}$ & 2 & & & \\
\hline & $\begin{array}{l}\text { Unweltpolitik ist nicht } \\
\text { besonders wichtig }\end{array}$ & 1 & & & \\
\hline \multicolumn{2}{|c|}{ Obligatorisches Potenzial } & & & $0-10 / / 0-1$ & 0,6 \\
\hline \multirow[t]{2}{*}{ Art des Gesetzes } & Supranationales Recht & 3 & $0-6$ & & \\
\hline & $\begin{array}{l}\text { Internationales "hard law" } \\
\text { Internationales "soft law" }\end{array}$ & $\begin{array}{l}2 \\
1\end{array}$ & & & \\
\hline \multirow[t]{5}{*}{ Monitoring } & Öberwachung durch & 4 & $0-4$ & & \\
\hline & Gerichtshöfe & 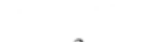 & & & \\
\hline & $\begin{array}{l}\text { Spezialisiertes } \\
\text { Überwachungsorgan }\end{array}$ & 3 & & & \\
\hline & Rechenschaftslegung & 2 & & & \\
\hline & Diplomatie & 1 & & & \\
\hline \multicolumn{2}{|c|}{ Kommunikatives Potenzial } & & & $0-40 / / 0-1$ & 0,8 \\
\hline $\begin{array}{l}\text { Häufigkeit der } \\
\text { Interaktion }\end{array}$ & $\begin{array}{l}\text { Zahl der jährlichen Treffen } \\
\text { der nationalen Vertreter }\end{array}$ & $(1 / 3-150)$ & $0-10$ & & \\
\hline $\begin{array}{l}\text { Anzahl der Orga- } \\
\text { nisationsorgane }\end{array}$ & $\begin{array}{l}\text { Organisationsorgane für } \\
\text { nationale Vertreter }\end{array}$ & $(0-10)$ & $0-10$ & & \\
\hline $\begin{array}{l}\text { Beständigkeit der } \\
\text { Repräsentation }\end{array}$ & $\begin{array}{l}\text { Exist enz einer dauerhaften } \\
\text { nationalen Vertretung }\end{array}$ & $(0 / 1)$ & $0-10$ & & \\
\hline $\begin{array}{l}\text { Anzahl der } \\
\text { Mitarbeiter }\end{array}$ & $\begin{array}{l}\text { Anzahl ständiger Mitarbeiter } \\
\text { in Umweltabteilungen }\end{array}$ & $(0-574)$ & $0-10$ & & \\
\hline \multicolumn{6}{|c|}{ Wirtschaftliche Verflechtung } \\
\hline $\begin{array}{l}\text { Bilaterale } \\
\text { Handelsoffenheit }\end{array}$ & $\begin{array}{l}\left(\text { Exporte }_{\mathrm{i} \rightarrow \mathrm{j}}+\text { Exporte }_{\mathrm{j} \rightarrow \mathrm{i}}\right) / \\
\text { total } \mathrm{BIP}_{\mathrm{i}<\mathrm{j}} \\
\forall \mathrm{i}, \mathrm{j} \ni\{\mathrm{WTO}, \text { Markt }\}\end{array}$ & $0-40964$ & & & \\
\hline
\end{tabular}




\begin{tabular}{|llccc|}
\hline Indikator & \multicolumn{1}{c}{ Kategorien } & Wert & Skala & \multicolumn{2}{c|}{$\begin{array}{c}\text { Aggregation } \\
\text { Index // } \\
\text { normalisiert }\end{array}$} & $\begin{array}{c}\text { Cronbachs } \\
\alpha\end{array}$ \\
\hline Kontrollvariablen & & & & 0,7 \\
\hline Kulturelle & Gemeinsame Grenze & $(0 / 1)$ & $0-3$ & \\
Ähnlichkeit & Gemeinsame Sprache & $(0 / 1)$ & & \\
& Gemeinsame Religion & $(0 / 1)$ & & \\
Einkommens- & BIP in US \$ & $1158-45496$ & & \\
niveau & Wahlerfolg grüner Parteien & $(0 / 1)$ & $0-3$ & \\
Politische & Mitgliedschaft im Parlament & $(0 / 1)$ & & \\
Nachfrage & Teilhabe an der Regierung & $(0 / 1)$ & & \\
Bevölkerungs- & Personen/km ${ }^{2}$ & $13-466$ & & \\
dichte & & & & \\
CO $_{2}$-Emissionen & Tonnen pro Kopf & $2-22$ & & \\
\hline
\end{tabular}




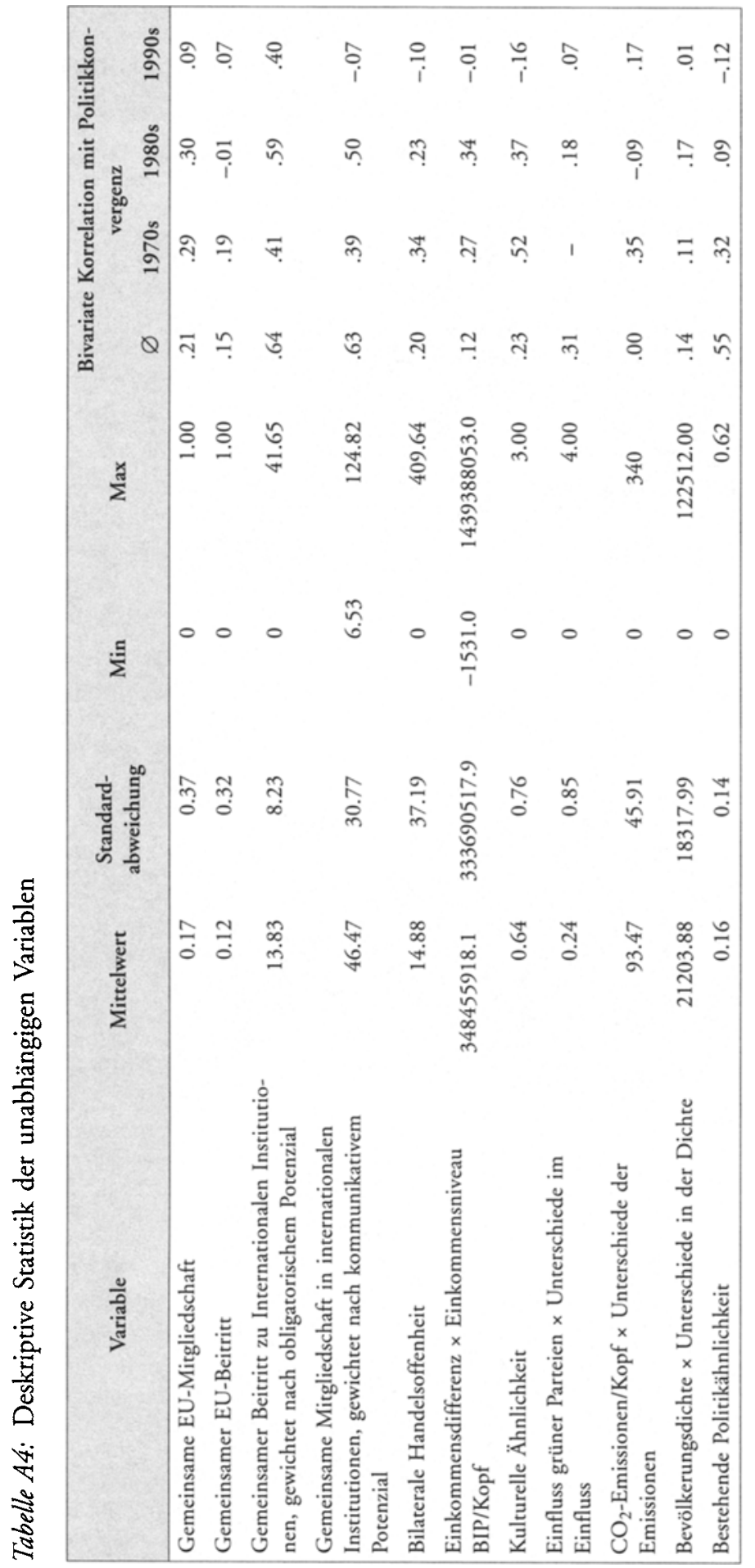

